\title{
ANÁLISIS DE LA LITERATURA SOBRE PLANTAS MEDICINALES EN COSTA RICA (1930-2001)
}

\author{
Mildred García-González ${ }^{1}$ \& Carlos O. Morales $^{2}$ \\ 1 Departamento de Fisiología, Escuela de Medicina, Universidad de Costa Rica, \\ 2 Escuela de Biología, Universidad de Costa Rica, 2060 San José, Costa Rica.
}

\begin{abstract}
A total of 416 publications on medicinal plants from Costa Rica are compiled from the period 19302001. These publications are classified and analysed according to publication date, kind, thematics, and names of plant families and genera with the greatest numbers of studies. The decade with most publications is the 1990 's (122 works, $29 \%$ of total), and 217 works (52\%) of the revised literature correspond to investigations made by students of the University of Costa Rica in the frame of graduate studies. Phytochemistry, with 292 works, represents $70 \%$ of the studies on medicinal plants. Asteraceae is the plant family with the greatest number of studies (47); the most studied genera are Citrus (Rutaceae) and Quassia (Simaroubaceae), both with 14 references.
\end{abstract}

RESUMEN. Se recopilan 416 publicaciones acerca de plantas medicinales en Costa Rica, en el período comprendido entre 1930 y 2001. Se clasifican y analizan estas publicaciones según fecha de publicación, tipo de publicación, temática y nombres de las familias y los géneros con mayor número de estudios. La década de mayor producción en este campo es la de 1990, con 122 trabajos (29\% del total). El 52\% (217 trabajos) de la literatura revisada corresponde a aportes de estudiantes de la Universidad de Costa Rica realizados mediante trabajos de graduación. La fitoquímica, con 292 trabajos, abarca el 70\% de la investigación de las plantas medicinales. La familia de plantas con mayor número de estudios (47) es Asteraceae y los géneros con el mayor número de trabajos científicos (14) son Citrus (Rutaceae) y Quassia (Simaroubaceae).

Palabras Clave / Kew Words: Plantas medicinales, estudios fitoquímicos, etnobotánica, Costa Rica

\section{INTRODUCCIÓN}

Las plantas son una alternativa actual para buscar nuevos agentes terapéuticos. De hecho, han sido utilizadas desde tiempos remotos con fines curativos, ya que uno de los anhelos de los seres humanos ha sido siempre intentar combatir sus enfermedades. Para ello, han hecho uso de organismos y productos que la naturaleza ofrece. Los usos particulares se han transmitido en forma oral o escrita y de generación en generación a lo largo de la historia, hasta nuestros días.

Muchas de las aplicaciones que se hacen de las plantas medicinales se relacionan con prácticas mágico-religiosas. A menudo, el resultado ha sido una sobrevaloración de ciertas plantas medicinales; es decir, asignarles una multitud de propiedades que en realidad no poseen.

En la actualidad existe un interés creciente por el estudio y la utilización de las plantas medicinales, tanto en países desarrollados como en aquéllos en desarrollo. No obstante, las causas principales son diferentes en cada uno de ellos. En países desarrollados se trata, a priori, de una moda, la cual intenta combatir el excesivo consumo de fármacos de síntesis o evitar los efectos secundarios que de ellos se derivan. En cambio, en los países en desarrollo se trata más bien de un problema socioeconómico, ya que una gran parte de la población no cuenta con los recursos económicos necesarios para una terapia farmacológica. Tanto en unos países como en otros, el resultado del uso de plantas medicinales ha sido, en muchas ocasiones, el abuso de drogas de origen vegetal, utilizadas con fines supuestamente terapéuticos. Sin embargo, en la mayoría de los casos no existe comprobación científica de la utilidad de los productos vegetales. Esta situación, existente en la mayoría de los países latinoamericanos, revela la importancia de validar científicamente las plantas medicinales de uso tradicional y popular, cuando realmente corresponde.

A partir de la década de 1940 el desarrollo económico de las plantas medicinales se ha caracterizado, en todo el mundo, por la identificación, la selección y el procesamiento de recursos vegetales para la elaboración de fármacos. El siguiente paso fue la síntesis química de principios activos con actividad farmacológica, de modo que los productos naturales dejaron de ser la fuente principal de los fármacos o medicamentos.

A medida que, primero, se realicen nuevos ensayos 
fitoquímicos y farmacológicos y, segundo, se validen o se rechacen los efectos atribuidos a ciertas plantas, muchos nombres desaparecerán de las listas actuales de plantas medicinales y otros nombres se agregarán.

La etnobotánica, o estudio del uso de las plantas en las sociedades tradicionales, ofrece grandes posibilidades para descubrir nuevos productos, útiles para la humanidad, derivados de las plantas. Muchos de los extractos de plantas utilizados por la medicina occidental se descubrieron porque ya se empleaban en sociedades tradicionales, aunque no siempre con el mismo fin. Sin embargo, también es importante aprovechar la etnobotánica para ayudar a las comunidades locales a adaptarse a las nuevas circunstancias ${ }^{1}$.

El estudio sistemático de la flora de Costa Rica se inició en la primera mitad del siglo XIX, sobre todo después de la llegada al país del naturalista danés Anders Sandoe Oersted en $1846^{2}$. Empero, los estudios científicos de las plantas medicinales se iniciaron mucho tiempo después, a partir de la década de 1930 , especialmente en las décadas posteriores a la fundación de la Universidad de Costa Rica en 1940.

El objetivo del presente estudio es realizar un análisis de la literatura sobre plantas medicinales y otras plantas útiles en Costa Rica, entre 1930 y 2001, como base para realizar nuevos estudios, verificar resultados o ampliarlos y, de este modo, evitar la duplicación de algunas investigaciones y aprovechar de manera más eficaz los recursos vegetales.

\section{Metodología}

Se inició una recopilación de literatura localizada en las bibliotecas de la Universidad de Costa Rica (UCR) y se realizó un esfuerzo por localizar bibliografía generada por otros centros de estudios costarricenses, como bibliotecas de la Universidad Nacional (UNA), del Instituto Tecnológico de Costa Rica (ITCR) y del Centro Agronómico Tropical de Investigación y Enseñanza (CATIE). Se citan algunos artículos de divulgación publicados en la prensa nacional, material audiovisual y algunas fuentes halladas en bibliotecas privadas. Se abarcó el período que va desde 1930 hasta inicios del año 2001. Se tomaron en cuenta especialmente trabajos de graduación, que luego fueron publicados como artículos en revistas científicas.

Además de los trabajos de toxicología y validación farmacológica de las plantas medicinales, se consideraron investigaciones fitoquímicas con algún potencial en plantas medicinales y trabajos sobre el estudio de la composición alimenticia de plantas, sus frutos y sus derivados (bromatología). En algunos casos se trata de estudios fitoquímicos de plantas útiles, que no son alimenticias y no necesariamente se usan en medicina popular; tal es el caso del mangle (Rhizophora mangle L. y otras especies del género, Rhizophoraceae; ver adelante referencias 25, 95 y 151). Su inclusión aquí se justifica ampliamente por tratarse de estudios de composición química de plantas.

Las publicaciones se clasificaron con base en los criterios siguientes:

1. Fecha de publicación. Se distinguen las siete décadas entre los años 1930 y 2001.

2. Tipo de publicación. Se distinguen aquí seis criterios: a) tesis universitarias de licenciatura, b) tesis universitarias de maestría, c) artículos científicos, publicados en revistas tanto nacionales como extranjeras, d) libros, e) memorias de actividades y f) prácticas dirigidas y videocintas.

3. Temática de las publicaciones. Se clasifican los trabajos recopilados en seis áreas, según el tema de la investigación realizada o la disciplina de estudio: a) estudios fitoquímicos, b) estudios farmacológicos, c) estudios fitoquímicos y farmacológicos, d) estudios bromatológicos, e) trabajos de divulgación etnomédica y f) comercialización de plantas medicinales.

4. Familias y géneros de plantas. Se recopila información acerca del número de trabajos realizados según la familia y el género.

\section{RESULTADOS Y DiscusióN}

El Cuadro 1 muestra la distribución de los trabajos por décadas desde 1930 hasta 2001.

Cuadro 1. Número de publicaciones sobre plantas medicinales en Costa Rica por décadas. 1930 - 2001.

\begin{tabular}{|c|c|c|}
\hline Período & $\begin{array}{c}\text { No. de } \\
\text { publicaciones }\end{array}$ & Porcentaje \\
\hline $1930-1939$ & 2 & 0,48 \\
\hline $1940-1949$ & 33 & 7,93 \\
\hline $1950-1959$ & 68 & 16,34 \\
\hline $1960-1969$ & 61 & 14,66 \\
\hline $1970-1979$ & 26 & 6,24 \\
\hline $1980-1989$ & 77 & 18,50 \\
\hline $1990-1999$ & 122 & 29,32 \\
\hline $2000-2001$ & 27 & 6,49 \\
\hline Total & 416 & 100 \\
\hline
\end{tabular}

1. Unión Internacional para la Conservación de la Naturaleza (UICN), 1993, Gland, Suiza.

2. Denyer, C.P. \& Soto, B.G. 1999. Contribución pionera de William M. Gabb a la geología y cartografía de Costa Rica. Anu. Estud. Centroamer. 25(2): 103-138. 
A partir de 1970 se dio en Costa Rica la universalización de la atención de la salud mediante la unificación de hospitales, la atención médica ambulatoria y el desarrollo de programas de atención primaria. Además, se elaboró un Plan Nacional de Salud, en el cual se destacaban como prioridades la atención del embarazo y del parto, la lactancia materna y el control y la erradicación de enfermedades prevenibles mediante vacunación (diarreas y parasitosis, infecciones respiratorias agudas y desnutrición) ${ }^{3}$. En esa década también se dio en el país un aumento en la importación de medicamentos, lo que posiblemente contribuyó a desviar el interés por el estudio científico de la medicina tradicional. Ese interés se recuperó a finales de la década de 1980 y se mantiene hasta el presente. Esto último se refleja en un aumento notable del número de publicaciones sobre plantas medicinales en la década de 1990.

En total, se recopilaron 220 trabajos universitarios de graduación, de los cuales el 94,54\% (208) corresponde a trabajos de licenciatura; de éstos, el 79,80\% (166) fueron realizados por estudiantes de farmacia. Este valor corresponde al $40 \%$ de la literatura revisada (Cuadro 2).

Cuadro 2. Tipos de publicaciones sobre plantas medicinales en Costa Rica. 1930-2001.

\begin{tabular}{|l|c|c|}
\hline \multicolumn{1}{|c|}{ Tipo de publicación } & $\begin{array}{c}\text { No. de } \\
\text { trabajos }\end{array}$ & Porcentaje \\
\hline Tesis de Licenciatura en Farmacia (UCR ${ }^{4}$ ) & 165 & 39,66 \\
Tesis de Licenciatura en Farmacia (UNIBE ${ }^{5}$ ) & 1 & 0,24 \\
\hline Tesis de Licenciatura en Química (UCR) & 28 & 6,73 \\
Tesis de Licenciatura en Ingeniería Química (UCR) & 5 & 1,20 \\
\hline Tesis de Licenciatura en Tecnología de Alimentos (UCR) & 7 & 1,68 \\
\hline Tesis de Licenciatura en Biología (UCR) & 2 & 0,48 \\
\hline Tesis de Maestría en Ciencias Biomédicas (UCR) & 8 & 1,92 \\
\hline Tesis de Maestría en Química (UCR) & 2 & 0,48 \\
\hline Prácticas dirigidas & & \\
- Licenciatura en Agronomía (UCR) & 1 & 0,24 \\
- Especialidad en Ingeniería Forestal (ITCR ${ }^{6}$ ) & 1 & 0,24 \\
\hline Revistas nacionales e internacionales & 136 & 32,69 \\
\hline Libros & 42 & 10,09 \\
\hline Memoria de actividades & 13 & 3,12 \\
\hline Otros & 5 & 1,20 \\
\hline Total & 416 & 100 \\
\hline
\end{tabular}

En la edad moderna, originalmente el conocimiento de las propiedades curativas de las plantas medicinales estuvo en poder de los médicos. A medida que fueron surgiendo medicamentos de síntesis y progresó la industria farmacéutica, este conocimiento fue abandonado paulatinamente por la medicina y retomado por los farmacéuticos, quienes hoy día siguen impartiendo en sus carreras el tema de la botánica aplicada o farmacognosia. Como muestra el presente análisis bibliográfico, en Costa Rica la farmacia, con la química, genera la mayor parte de la información sobre la flora medicinal.

Es interesante notar que muchos de los trabajos realizados en el país han sido publicados en revistas, tanto nacionales como internacionales. Sin embargo, la mayoría de los trabajos de graduación quedan archivados en las bibliotecas; esto motiva un llamado de atención a las entidades educativas superiores, que deben facilitar la publicación de estos trabajos para que lleguen a la comunidad científica, se evite la duplicidad de esfuerzos y la pérdida de información valiosa y recursos económicos.

El número de libros no es despreciable, ya que corresponde al 10,09\% del total de la literatura compilada. Estos libros no son, en su mayoría, de carácter estrictamente científico; se trata más bien de información sobre uso popular de plantas medicinales. Algunos se refieren al uso tradicional basado en la 
transmisión oral de generación en generación, otros al cultivo y la agrotecnología de plantas medicinales. En uno de ellos (referencia \# 131, ver adelante) se analizan las legislaciones iberoamericanas para la inscripción sanitaria, en los ministerios de salud, de productos farmacéuticos con base en plantas medicinales. La información recopilada en los libros es rescatable, por cuanto contiene parte de la idiosincrasia costarricense en materia de conocimiento del uso tradicional de plantas medicinales, que paulatinamente ha ido desapareciendo en las nuevas generaciones.

La literatura también se clasificó según la disciplina que generó el conocimiento, lo cual se presenta en el Cuadro 3.

Cuadro 3. Número de trabajos sobre plantas medicinales en Costa Rica, según la disciplina de estudio desarrollada. 1930-2001.

\begin{tabular}{|l|c|c|}
\hline \multicolumn{1}{|c|}{ Temática } & $\begin{array}{c}\text { No. de } \\
\text { trabajos }\end{array}$ & Porcentaje \\
\hline Estudios fitoquímicos & 202 & 48,56 \\
\hline Estudios fitoquímicos y farmacológicos & 65 & 15,63 \\
\hline Estudios fitoquímicos y bromatológicos & 25 & 6,00 \\
\hline Estudios bromatológicos & 14 & 3,37 \\
\hline $\begin{array}{l}\text { Estudios farmacológicos } \\
\text { (ie., actividad de las plantas medicinales) }\end{array}$ & 10 & 2,40 \\
\hline Divulgación del conocimiento etnofarmacológico y otros & 84 & 20,19 \\
\hline Comercialización & 16 & 3,85 \\
\hline Total & 416 & 100 \\
\hline
\end{tabular}

Los estudios fitoquímicos incluyen la búsqueda e identificación de principios activos o característicos de las plantas; los trabajos farmacológicos se ocupan de las acciones farmacológicas y sus posibles mecanismos; las obras de divulgación (libros y artículos) recopilan el conocimiento popular sobre la acción de las plantas medicinales.

Existe una gran diferencia entre el conocimiento generado por la fitoquímica y el de otros campos del saber. Esto se comprende al tomar en cuenta la mayor y mejor estandarización de los ensayos fitoquímicos, además de la menor complejidad para determinar compuestos químicos básicos. Los estudios farmacológicos resultan, en la mayoría de los casos, más caros y laboriosos; requieren de técnicas y equipos más especializados, que no siempre están al alcance de los grupos de investigación. Además, es necesario contar con el reactivo biológico, que es el animal de laboratorio con el cual se realiza la investigación preclínica de la posible toxicidad de los productos naturales y la eventual validación del efecto farmacológico atribuido por la población. En el presente todavía siguen faltando metodologías adecuadas para la investigación científica de plantas medicinales.

El análisis bibliográfico revela el estudio de 123 familias de plantas, de las cuales $33(26,8 \%)$ poseen una sola referencia y $21(17,07 \%)$ poseen 10 o más estudios. En el Cuadro 4 se presentan las 11 familias con los mayores números de trabajos realizados y el número de géneros que se han estudiado en cada una de estas familias. El género con mayor número de estudios de la familia Asteraceae es Matricaria, con 10 trabajos tanto fitoquímicos como farmacológicos (referencias 32, 40, 43, 44, 46, 126, 128, 154, 164 y $180)$; los géneros de esta familia con un solo trabajo son Cichorium (ref. 63), Melampodium (ref. 101), Podachaenium (ref. 65), Tanacetum (ref. 337) y Tithonia (ref. 338).

En promedio, existen solamente dos trabajos por género. La familia Rutaceae posee el mayor índice de estudios por género $(4,17)$, ya que 25 referencias tratan sobre seis géneros; de éstos, Citrus es el que tiene mayor número de trabajos (en total 14 referencias, Cuadro 5), mientras que Galipea (ref. 183) y Pilocarpus (ref. 307) solamente tienen una referencia. Rubiaceae es la familia con el menor índice de estudios por género $(0,52)$, porque 16 referencias abarcan 31 géneros.

De un total de 402 géneros, 229 (56,9\%) tienenuna sola referencia. En el Cuadro 5 se presentan los 11 géneros con mayores números de estudios y sus respectivas familias. Esto evidencia la gran heterogeneidad del trabajo científico realizado con la flora medicinal en Costa Rica. Además, en la lista de referencias incluida adelante, ordenada alfabéticamente por nombres de autores, se nota una ausencia de continuidad en los trabajos de investigación, ya que muchos de los 
estudios realizados son preliminares o parciales y carecen de componentes de interdisciplinariedad, que son indispensables para asegurar el éxito del estudio científico de las plantas medicinales. Además, ciertos trabajos muestran duplicidad de información; esto implica que se han repetido investigaciones que otros ya habían realizado en el pasado. Asimismo, una parte de la información está duplicada, porque se citan tanto tesis originales como artículos de revistas científicas derivados de esas tesis.

Cuadro 4. Familias con mayores números de estudios sobre plantas medicinales y número de géneros estudiados en cada una. Costa Rica: 1930-2001.

\begin{tabular}{|c|c|c|c|c|}
\hline Familia & $\begin{array}{l}\text { No. de } \\
\text { géneros } \\
\text { estudiados }\end{array}$ & $\begin{array}{l}\text { No. de } \\
\text { trabajos }\end{array}$ & $\begin{array}{l}\text { Porcentaje } \\
\text { del total de } \\
\text { trabajos }\end{array}$ & Referencias \\
\hline 1. Asteraceae & 18 & 47 & 11,30 & $\begin{array}{l}3-21-26-32-40-43-44-46-54-59-63-64-65-86- \\
99-101-104-112-126-128-139-140-141-142- \\
154-164-168-180-196-208-224-233-234-301- \\
320-321-323-325-326-327-328-329-332-333- \\
337-345-393 .\end{array}$ \\
\hline 2. Fabaceae & 17 & 37 & 8,78 & $\begin{array}{l}\text { 5-12-32-101-118-139-141-144-145-148-149- } \\
\text { 166-167-177-181-185-216-220-231-243-245- } \\
\text { 285-397-306-311-320-324-340-349-345-358- } \\
361-369-371-386-393-407 .\end{array}$ \\
\hline 3. Apocynaceae & 9 & 25 & 5,93 & $\begin{array}{l}\text { 43-52-79-81-83-84-85-103-106-110-137139- } \\
\text { 140-141-221-244-250-335-347-341-342-345- } \\
377-384-385 \text {. }\end{array}$ \\
\hline 4. Rutaceae & 6 & 25 & 5,93 & $\begin{array}{l}\text { 4-32-39-43-101-117-136-131-129-129-170- } \\
\text { 174-183-209-245-288-289-307-345-349-380- } \\
\text { 388-389-393-404. }\end{array}$ \\
\hline 5. Euphorbiaceae & 18 & 24 & 5,70 & $\begin{array}{l}\text { 2-32-101-110-113-116-125-139-140-141-195- } \\
\text { 207-237-238-239-245-268-287-345-349-351- } \\
\text { 360-393-408. }\end{array}$ \\
\hline 6. Solanaceae & 9 & 19 & 4,51 & $\begin{array}{l}7-38-43-51-53-100-101-109-123-127-190-205- \\
207-245-295-296-349-356-393 .\end{array}$ \\
\hline 7. Myrtaceae & 6 & 17 & 4,03 & $\begin{array}{l}29-32-55-94-101-169-172-245-268-331-349- \\
345-375-374-373-393-398\end{array}$ \\
\hline 8. Rubiaceae & 31 & 16 & 3,80 & $\begin{array}{l}\text { 67-101-112-138-182-207-240-245-251-268- } \\
277-293-349-345-386-393\end{array}$ \\
\hline 9. Lauraceae & 5 & 16 & 3,80 & $\begin{array}{l}\text { 68-101-156-184-245-284-313-315-318-319- } \\
336-345-349-371-372-393\end{array}$ \\
\hline 10. Moraceae & 8 & 16 & 3,80 & $\begin{array}{l}16-101-141-245-268-290-294-312-317-345- \\
348-349-354-393-403-412\end{array}$ \\
\hline
\end{tabular}


Cuadro 5. Géneros de plantas medicinales con mayores números de estudios en Costa Rica. 1930-2001.

\begin{tabular}{|c|c|c|c|c|}
\hline Género & Familia & $\begin{array}{l}\text { Número de } \\
\text { estudios }\end{array}$ & Porcentaje & Referencias \\
\hline 1. Citrus & Rutaceae & 14 & 3,33 & $\begin{array}{l}4-32-39-101-117-174-209- \\
288-289-380-388-389-393- \\
404 .\end{array}$ \\
\hline 2. Quassia & Simaroubaceae & 14 & 3,33 & $\begin{array}{l}27-32-43-134-202-203-215- \\
257-255-253-345-393-399- \\
402 .\end{array}$ \\
\hline 3. Piper & Piperaceae & 12 & 2,61 & $\begin{array}{l}\text { 69-88-89-101-109-123-146- } \\
216-168--225-339-393 .\end{array}$ \\
\hline 4. Smilax & Smilacaceae & 11 & 2,61 & $\begin{array}{l}32-60-101-143-176-222-242- \\
252-268-393-401 .\end{array}$ \\
\hline 5. Stemmadenia & Apocynaceae & 11 & 2,61 & $\begin{array}{l}\text { 79-81-84-85-137-139-140- } \\
221-341-377-384 .\end{array}$ \\
\hline 6. Cassia & Fabaceae & 10 & 2,38 & $\begin{array}{l}32-99-129-138-180-216-310- \\
322-349-406 .\end{array}$ \\
\hline 7. Gliricidia & Fabaceae & 10 & 2,38 & $\begin{array}{l}101-139-140-166-177-231- \\
358-361-370-393 .\end{array}$ \\
\hline 8. Matricaria & Asteraceae & 10 & 2,38 & $\begin{array}{l}32-40-43-44-46-126-128-154- \\
164-180 .\end{array}$ \\
\hline 9. Musa & Musaceae & 9 & 2,14 & $\begin{array}{l}\text { 96-98-101-107-173201-379- } \\
382-393 .\end{array}$ \\
\hline 10. Siparuna & Monimiaceae & 9 & 2,14 & $\begin{array}{l}30-139-140-188-189-193-245- \\
349-345 .\end{array}$ \\
\hline 11. Solanum & Solanaceae & 9 & 2,14 & $\begin{array}{l}8-100-101-109-123-127-205- \\
245-296 .\end{array}$ \\
\hline
\end{tabular}

\section{CONCLUSIONES Y RECOMENDACIONES}

La presente investigación revela una diferencia muy notable entre el número de estudios fitoquímicos (202) y el de exclusivamente farmacológicos (10). Esta diferencia podría deberse a que los estudios fitoquímicos están, desde hace algún tiempo, mejor estandarizados y utilizan en muchas ocasiones técnicas menos complejas para la identificación de algunos compuestos característicos. Los estudios 
farmacológicos resultan ser, con frecuencia, más caros y laboriosos, y requieren, además, del uso de animales de laboratorio, de técnicas y equipo más especializados, que no siempre están al alcance de todos los investigadores. Lamentablemente, esta diferencia en el quehacer fitoquímico y farmacológico se mantiene hasta nuestros días.

Los resultados del presente trabajo también muestran que las investigaciones sobre plantas medicinales se realizan con una orientación independiente y aislada de otros intereses académicos. Por ello, es necesario realizar investigaciones sobre plantas medicinales con un enfoque interdisciplinario; para lograrlo, deben involucrarse diferentes disciplinas asociadas, que puedan conducir a metas concretas de interés común.

Los estudios farmacológicos deben fortalecerse y aprovecharse, con el propósito de contar con criterios científicos para el uso seguro de las plantas medicinales, tanto en los niveles popular y nacional como industrial e internacional.

Algunas personas siguen utilizando y recomendando muchas especies de plantas, incluso sin tener ninguna comprobación del efecto que se les atribuye, o después de haberse descartado cualquier efecto positivo mediante ensayos farmacológicos. En algunos casos, se han comunicado hasta efectos secundarios no deseables de algunas plantas de uso común. En general, los usuarios de plantas medicinales se basan en el refrán popular que reza "las plantas, si no curan, no hacen daño". Ahora sabemos que algunas plantas de uso común pueden ser dañinas para la salud humana.

Un estudio serio sobre una especie medicinal debe, en primer lugar, hacer un análisis riguroso de las diferentes fracciones fitoquímicas que se obtienen, hasta encontrar el principio activo responsable de la acción atribuida a la planta; en segundo lugar, dilucidar los mecanismos de acción de los productos o extractos. El trabajo conjunto y multidisciplinario de los botánicos (identificación de plantas), los fitoquímicos (caracterización de componentes) y los farmacólogos (validación de la actividad atribuida) permitirá obtener resultados más sólidos y concluyentes.

Aún cuando todas las plantas medicinales tuvieran que clasificarse como inadecuadas para la salud, muchas de ellas seguirían empleándose en la medicina popular, ya que representan la única opción de salud disponible en muchos lugares del mundo. La Organización Mundial de la Salud (O.M.S.) reconoce que en los países subdesarrollados más de un tercio de la población no tiene acceso a los medicamentos esenciales; por ello, el uso de tratamientos tradicionales seguros y eficaces puede jugar un papel importante para mejorar la atención sanitaria?.

La vegetación del mundo se ha deteriorado $\mathrm{y}$ ha desaparecido a un ritmo alarmante. Muchas sociedades tradicionales han vivido en contacto directo con la naturaleza y han utilizado los productos de ésta para cubrir sus propias necesidades. Consecuentemente, estos grupos humanos tienen profundos conocimientos sobre las plantas locales, que en muchos casos también están a punto de perderse debido a los rápidos cambios culturales, sociales y económicos del mundo actual ${ }^{8}$. De este modo, queda clara la importancia de estudiar científicamente las plantas usadas tradicionalmente como medicinales, así como las múltiples especies vegetales silvestres que son desconocidas para la ciencia médica.

Agradecimientos. Al Laboratorio de Ensayos Biológicos de la Universidad de Costa Rica, a Kristhel García, Juan Alberto Rojas, Rosibel Vargas, Irene Gutiérrez, Karen Cordero, Ernesto Argüello, Nancy Rojas, Ivannia Rivas y Marcia Salas, por su invaluable trabajo en la recopilación de la información, y a Jaime García, del Centro de Educación Ambiental de la Universidad Estatal a Distancia (UNED), por la revisión del borrador de este trabajo y las sugerencias hechas. También a Bernal Morera (editor, Rev. Biol. Trop.) por sus comentarios críticos sobre una versión preliminar del escrito.

\section{NOTAS SOBRE LA LISTA DE REFERENCIAS}

No fue posible fijar un solo formato de citas bibliográficas, debido a la heterogeneidad de los escritos y a la diversidad de fuentes consultadas, que no siempre incluyen toda la información requerida por revistas científicas. Después de la lista de referencias se ofrece un índice de familias, géneros y especies estudiados.

7. O.M.S. 1991. Pautas para la evaluación de medicamentos herbarios. WHO/TRM/91.4. Programa de medicina tradicional. Ginebra. (original en inglés)

8. UICN. 1993. Loc. cit. \# 1. 


\section{LISTA DE REFERENCIAS SOBRE PLANTAS MEDICINALES Y OTRAS ESPECIES ÚTILES EN COSTA RICA (1930-2001)}

1.Acuña Arias, A. 1954. Análisis de la cera obtenida de Myrica. Tesis de Licenciatura en Farmacia, Universidad de Costa Rica.

2.Acuña Delgado, E. 1959. Investigación de la presencia de glicósidos cianogenéticos en la almendra tropical (Terminalia catappa), seso vegetal (Blighia sapida) y en la yuca amarga (Manihot utilissima). Tesis de Licenciatura en Farmacia, Universidad de Costa Rica.

3.Acuña Hidalgo, H. 1961. Ensayos biológicos del poder antiofídico de la Mikania guaco Humb. \& Bonpl. contra el veneno de la terciopelo (Bothrops atrox). Tesis de Licenciatura en Farmacia, Universidad de Costa Rica.

4.Acuña M., G. 1957. Estabilidad del ácido ascórbico en el Citrus sinensis con relación al tiempo y la temperatura. Tesis de Licenciatura en Farmacia, Universidad de Costa Rica.

5.Acuña Vargas, O. 1952. Comparación físico-química de la oleorresina de copaiba obtenida de la Prioria copaifera Griseb. de Costa Rica con el producto oficial de la F.E.U. XI. Tesis de Licenciatura en Farmacia, Universidad de Costa Rica.

6.Aguilar Ramírez, R. 2001. Apoyo técnico para el desarrollo sostenible de un grupo de mujeres productoras de plantas medicinales de El Cairo de Siquirres. Práctica dirigida de Licenciatura en Agronomía con énfasis en Fitotecnia, Facultad de Agronomía, Escuela de Fitotecnia, Universidad de Costa Rica.

7. Alfaro Lara, C.E. 1969. Glicoalcaloides esteroidales en especies costarricenses del género Solanum. Tesis de Licenciatura en Farmacia, Universidad de Costa Rica.

8. Alfaro Odio, R. 1960. Estudio del aceite esencial de la Gaultheria gracilis Small. Tesis de Licenciatura en Farmacia, Universidad de Costa Rica.

9.Alfaro Sagot, M. 1941. El tacaco. Tesis de Licenciatura en Farmacia, Universidad de Costa Rica.

10.Alfaro, A. 1931. Doscientas plantas medicinales de Costa Rica. Ciencia 19.

11.Almanza Mejía, E. 1961. Análisis bromatológico comparativo de los tubérculos de Xanthosoma violaceum (tiquisque) y Colocasia esculenta (ñampí) y su posible aplicación en la industria. Tesis de Licenciatura en Farmacia, Universidad de Costa Rica.

12.Amador, C. 1947. La jícama (Pachyrhizus angulatus Rich., Pachyrhizus erosus (L.) Urban). Tesis de Licenciatura en Farmacia, Universidad de Costa Rica.

13.Ammour, T., Ocampo, R. \& Robles, G. 1994. Caracterización de los sectores asociados a la producción, comercialización y transformación de plantas medicinales en Costa Rica. Documento de trabajo No. 3, CATIE. Turrialba.

14.Ampiée H., M. 1955. Investigación cualitativa de vitamina y protovitamina $\mathrm{A}$ en el achiote. Tesis de Licenciatura en Farmacia, Universidad de Costa Rica.

15.Ampiée H., M. 1956. Investigación cualitativa de vitamina A y provitamina A en el achiote, Bixa orellana L. Rev. Biol. Trop. 4(2): 227-233.

16.Araya R., M.E. 1966. Análisis químico bromatológico de las semillas del Artocarpus altilis. Tesis de Licenciatura en Farmacia, Universidad de Costa Rica.

17.Arguedas Barrantes, B. 1961. Estudio bromatológico del palmito de coyol (Acrocomia vinifera). Tesis de Licenciatura en Farmacia, Universidad de Costa Rica.

18.Arguedas Troyo, M.A. 1961. Contribución al estudio del sainillo (Dieffenbachia seguine). Tesis de Licenciatura en Farmacia, Universidad de Costa Rica.

19.Arias Avendaño, M. 1994. Obtención de un producto deshidratado a partir de cebolla (Alliun сера). Tesis de Licenciatura en Tecnología de Alimentos, Universidad de Costa Rica.

20.Arias Herrera, A.Y. \& Arias Monteverde, M. 1996. Verificación de la actividad anti-inflamatoria, determinación de la actividad analgésica y del modo de acción del extracto acuoso bruto de Loasa speciosa (Loasaceae). Tesis de Licenciatura en Farmacia, Universidad de Costa Rica.

21.Artavia Vargas, M. 1945. El tuete (Vernonia canescens H.B.K.). Tesis de Licenciatura en Farmacia, Universidad de Costa Rica.

22.Atán Chan, J. 1996. Identificación de algunos compuestos tóxicos y análisis microbiológico de plantas medicinales empacadas para el consumo humano. Tesis de Licenciatura en Farmacia, Universidad de Costa Rica.

23.Aved Gottos, C. 1961. Ensayo fitoquímico de la especie Vincetoxicum edule (cuayote). Tesis de Licenciatura en Farmacia, Universidad de Costa Rica. 
24.Ávila, A. M., Barquero, L. \& Calzada, J. 1982. Una mejora en el método de determinación de bixina en achiote. Ing. Cienc. Química 6(3-4): 209-210.

25.Avram Waganoff, M. 1976. Estudio comparativo del extracto de mangle en curtición ultra-rápida. Tesis de Licenciatura en Química, Universidad de Costa Rica.

26.Badilla Baltodano, B. 1995. Validación y búsqueda de actividad antiinflamatoria en plantas medicinales de la flora costarricense. Tesis de Maestría en Ciencias Biomédicas, Universidad de Costa Rica.

27.Badilla, Miranda, Mora \& Vargas. 1998. Actividad gastrointestinal del extracto acuoso bruto de Quassia amara (Simaroubaceae). Rev. Biol. Trop. 46: 203.

28.Badilla, B., Mora, G., Lapa, J. \& Silva, A. 1999. Anti-inflamatory activity of Urera baccifera (Urticaceae) in Sprague-Dawley rats. Rev. Biol. Trop. 47: 365 .

29.Barboza R., D. 1952. Investigación de pepsina en la guayaba. Tesis de Licenciatura en Farmacia, Universidad de Costa Rica.

30.Barillas Porras, W. 1992. Contribución al estudio fitoquímico de la planta Siparuna griseo-flavescens. Tesis de Licenciatura en Farmacia, Universidad de Costa Rica.

31.Barrantes Mora, N. 1960. Ensayo químico bromatológico sobre el fruto comestible de la Bellucia costaricensis. Tesis de Licenciatura en Farmacia, Universidad de Costa Rica.

32.Barrientos, G. 1991. El uso y abuso de las plantas medicinales en la medicina tradicional costarricense. Biocenosis 7(2): 66-70.

33.Barrientos, G. 1992. Uso y abuso de las plantas medicinales, recetas y consejos al alcance de su mano. San José, Centro de Estudios y Publicaciones Alforja (CEPAL).

34.Barrios, M., Calvo, M., Arguedas, E. \& Castro, O. 1991. Epicatequina en Clusia stenophylla y Clusia flava. Ing. Cienc. Química 13(2): 27-28.

35.Barth, L. 1995. Valorando los productos forestales no maderables. Rev. For. Centroamer. (CATIE) 4(13): 35-40.

36.Beirute B., A. 1962. El repollo y sus efectos vermífugos. Tesis de Licenciatura en Farmacia, Universidad de Costa Rica.

37.Blanco de Pardo, N. 1956. Estudio de la Myristica fragrans. Tesis de Licenciatura en Farmacia, Universidad de Costa Rica.

38.Blanco Rodríguez, A.L. 1968. Determinación cuantitativa de los alcaloides de especies costarricenses de Datura arborea L. (reina de la noche). Tesis de Licenciatura en Farmacia, Universidad de Costa Rica.

39.Bolaños Arias, V. 1960. Obtención de pectina de residuos de frutos cítricos y sus posibilidades industriales en Costa Rica. Tesis de Licenciatura en Farmacia, Universidad de Costa Rica.

40.Bolaños Zamora, E.M. 2001. Determinación de las condiciones adecuadas de procesamiento de manzanilla seca (Chamomilla recutita) para la preparación de infusiones. Tesis de Licenciatura en Tecnología de Alimentos, Facultad de Agronomía, Universidad de Costa Rica.

41.Bonilla Campos, M.A. 1947. Análisis del fruto y mucílago de la corteza del guácimo (Guazuma ulmifolia, Esterculiáceas). Tesis de Licenciatura en Farmacia, Universidad de Costa Rica.

42.Bonilla, M.E. 1956. Estudio bromatológico del palmito (Euterpe longepetiolata). Tesis de Licenciatura en Farmacia, Universidad de Costa Rica.

43.Brenes, M. 1994. Las plantas curan pero también matan. La República. Suplemento Galería. p. 4 B, 26 de noviembre.

44.Brenes, M. 1994. Aromática y curativa manzanilla. La República. Suplemento Galería. p. 2 B, 6 de setiembre.

45.Brenes, M. 1994. En casa. Exótica frescura. La República, Suplemento Galería, p. 1B, 20 sept.

46.Brenes R., R., García, E. \& Hernández, E. 1962. Acción de algunos medicamentos sobre el músculo liso intestinal de conejo. O’Bíos 2: 34-37.

47.Brenes Vargas, L.D. \& Carranza González, A.M. 1999. Aportación al estudio de la solubilización micelar de aceites esenciales de Satureja viminea (Lamiaceae) y Lippia alba (Verbenaceae) y su efecto sobre la actividad antiinflamatoria tópica empleando un modelo animal. Tesis de Licenciatura en Farmacia, Universidad de Costa Rica.

48.Brenes, A. \& Campos, V. 1964. Compendio de la investigación científica realizada en la Facultad de Farmacia. Tesis de Licenciatura en Farmacia, Universidad de Costa Rica.

49.Brown Knight, C. 1958. Determinación de cianuro en algunas especies de nuestra flora. Tesis de Licenciatura en Farmacia, Universidad de Costa Rica.

50.Burgos C., M. 1940. El pejibaye. Tesis de Licenciatura en Farmacia, Universidad de Costa Rica.

51.Burgos Polit, M.A. 1968. Contenido alcaloidal de especies costarricenses de Datura stramonium L. (tapate). Tesis de Licenciatura en Farmacia, 
Universidad de Costa Rica.

52.Cabezas, J., \& Cicciò, J. 1986. Estudio químico de las hojas de Tabernaemontana arborea Rose. Ing. Cie. Quím. 10(3-4): 54.

53.Calderón Arias, M.E. 1960. Contribución al estudio del sulfatillo (Capsicum tetramerum Standl. \& Morton). Tesis de Licenciatura en Farmacia, Universidad de Costa Rica.

54.Calvo Brenes, G. 1984. Estudio fitoquímico de las hojas de la planta Montanoa hibiscifolia. Tesis de Licenciatura en Química, Universidad de Costa Rica.

55.Calvo Jara, R.A. 1948. Análisis y estudio del fruto del guayabo (Psidium guajava, Mirtáceas). Tesis de Licenciatura en Farmacia, Universidad de Costa Rica.

56.Carmona Sandí, A.T. \& Gutiérrez Ventura, Y. 1992. $\mathrm{Su}$ salud en el maravilloso mundo de las plantas. Rincón de Plantas. San José, Costa Rica.

57.Carranza Castro, R. 1950. Obtención, rendimiento y características de los aceites esenciales de zacate de limón y de zacate de violeta, cultivados en Costa Rica. Tesis de Licenciatura en Farmacia, Universidad de Costa Rica.

58.Castillo Umaña, M. 1963. Análisis químico bromatológico de Achras zapota L. (níspero o níspero de Guanacaste). Tesis de Licenciatura en Farmacia, Universidad de Costa Rica.

59. Castro Castillo, O. 1985. Estudio químico del aceite esencial de Tagetes microglossa Benth., familia Compositae. Ing. Cie. Quím. 9(3): 94-95.

60.Castro Castillo, O., Gutiérrez Gutiérrez, J.M., Villegas Aguilar, J.R., Robles Valle, G. \& Villalobos Soto, R. 1998. Evaluación de la actividad antihemorrágica de Smilax spp. Plantas medicinales del género Smilax en Centroamérica. Reuniones Técnicas; 22-25 sept. 1997, Actas de la reunión. CYTED; RIPROFITO. Serie Técnica (CATIE) No. 2, p. 147-148. Turrialba, Costa Rica.

61.Castro Castillo, O., Hoet, P. \& Poveda Álvarez, L.J. 1982. L'usage medicinal des plantes au Costa Rica. P1. Medic. Phytother. 16(3): 230-245.

62.Castro Gamboa, I. 1994. Evaluación fitoquímica de la parte aérea de Verbena litoralis HBK. Tesis de Licenciatura en Química, Universidad de Costa Rica.

63. Castro Lee, B. 1942. Apuntes sobre el calcio con referencia a determinaciones de ácido oxálico y calcio hechas en algunas legumbres costarricenses. Tesis de Licenciatura en Farmacia. Universidad de Costa Rica.

64.Castro, Mora, Jakupovic, Poveda \& Mata. 2000.
Nuevo estudio fitoquímico de Lycoseris latifolia, Asteraceae. Ing. Cie. Quím. 19: 15.

65.Castro, Murillo, Laas, Meunier, Mora, Pahl \& Merfort. 2000. Inhibition of the transcription factor NF-kn by sesquiterpene lactones from Podachaenium eminens (Asteraceae). Planta Med. 66: 591 .

66.Castro, O. \& Mora, G. 1982. Presencia de ácido $\alpha$-eleoesteárico en Fevillea cordifolia L., Cucurbitaceae. Ing. Cie. Química 6(2): 181-182.

67.Castro, O. \& López, J. 1986. Harman, alcaloide mayoritario de la raíz de Simira maxonii Standl., Rubiaceae. Ing. Cie. Quím. 10(3-4): 56-57

68.Castro, O. \& Muñoz, L. 1982. Alcaloides aporfínicos en hojas de Phoebe pittieri (familia Lauraceae). Ing. Cie. Quím. 6(3-4): 198-199.

69.Castro, O. \& Poveda, L. 1983. Piper auritum (H.B.K.), familia Piperaceae. Estudio preliminar del aceite esencial de sus hojas. Ing. Cie. Quím. 7(1-2): 24-25.

70.Castro, O., Durán, F. \& Chavarría, G. 1981. Estudio preliminar sobre la Verbena litoralis. Ing. Cie. Quím. 1(1): 13-14.

71.Castro, O. 1973. Estudio preliminar sobre la Verbena litoralis. Tesis de Licenciatura en Química, Universidad de Costa Rica.

72.Castro, V., Arias, R., Calzada, J. \& Poveda, L. 1982. Estudio preliminar de Zinowiewia costaricensis Lundell. Ing. Cie. Quím. 6(1): 156.

73.Castro, V., Mojica, E. \& Calzada, J. 1986. Tres nuevos diterpenos de Gyminda costaricensis Standl. Ing. Cie. Quím. 10(1-2): 1-6.

74.CEMPRO. 1983. Resultados del análisis de la situación del grupo de empresas exportadoras de plantas ornamentales, medicinales y semillas. San José, Costa Rica.

75.Centro de Investigación en Productos Naturales (CIPRONA). 1984. Agroindustrielle ontwikkeling van medicinale planten in Costa Rica: projectvoorstel. San José, Universidad de Costa Rica.

76.Cerdas Cruz, J. 1958. Contribución al estudio del aceite obtenido de las semillas de paste. Tesis de Licenciatura en Farmacia, Universidad de Costa Rica.

77.Cerdas Mora, F. 1948. Alantoína en el llantén. Tesis de Licenciatura en Farmacia, Universidad de Costa Rica.

78.Cerna Castro, A.M. \& Alvarado, M. (entrevistador). 1998. La naturaleza tiene el remedio. Grabaciones Sonoras. San Ramón, Alajuela, Sede de Occidente, Universidad de Costa Rica. 
79.Cicciò A., J.F. 1973. Alcaloides de Stemmadenia glabra Benth. Tesis de Licenciatura en Química, Universidad de Costa Rica.

80.Cicciò A., J.F. 1977. Estudio físico y químico de un aceite esencial de cardamomo guatemalteco. Cie. Tec. 1(1): $5-12$.

81.Cicciò, J.F. 1977. Tabersonina y coronaridina en semillas de plantas del género Stemmadenia (Apocynaceae). Brenesia 12/13: 21-24.

82.Cicciò, J.F. 1984. Poligodiol, constituyente mayoritario de la corteza de Drimys granadensis L.f. (Winteraceae). Ing. Cie. Quím. 8(3-4): 45-46.

83.Cicciò, J., Castro, V. \& Urbina, A. 1985. Alcaloides indólicos de las ramitas de Tabernaemontana arborea Rose. Ing. Cie. Quím. 9(4): 133-135.

84.Cicciò, J.F. \& Chavarría, R.G. 1974. Estudio fitoquímico de Stemmadenia glabra Benth. (Apocynaceae). I. Análisis alcaloidal. Brenesia 4: 1- 3.

85.Cicciò, J.F. \& Guzmán, O.M. 1976. Aceites de semillas de plantas del género Stemmadenia (Apocynaceae). Brenesia 9: 31.

86.Cicciò, J.F., Calzada, J., Montero, W. \& Poveda, L. 1978. Estudio fitoquímico preliminar de plantas de la familia Compositae de Costa Rica. Rev. Biol. Trop. 26(1): 159-165.

87.Cicciò, Soto \& Poveda. 1999. Essential oil of Lepechinia schiedeana (Lamiaceae) from Costa Rica. Rev. Biol. Trop. 47(3): 377.

88.Cicciò, J.F. \& Segnini, M. 1998. Essential oil of leaves and spikes of Piper peltatum L. from Costa Rica. Ing. Cie. Quím. 18(2): 55.

89.Cicció, J.F. \& Ballestero, C. 1997. Constituyentes volátiles de las hojas y espigas de Piper aduncum (Piperaceae) de Costa Rica. Rev. Biol. Trop. 45(2): 783.

90.Cordero Rojas, F. 1963. Estudio químico bromatológico de las hojas y parte del tallo comestible del bledo blanco, Amaranthus hybridus. Tesis de Licenciatura en Farmacia, Universidad de Costa Rica.

91.Cordero, M. \& Carvajal, A. 1953. Los cogollos del jocote como antianémico. Tesis de Licenciatura en Farmacia, Universidad de Costa Rica.

92.Córdoba Espinoza, L. 1963. Acción de las hormonas vegetales sintéticas 2-4-D y 2-4-5T sobre Streptococcus y Staphylococcus. Tesis de Licenciatura en Farmacia, Universidad de Costa Rica.

93.Córdoba Murillo, F. 1999. Evaluación de la inhibición del edema y de la hemorragia inducidos por el veneno de Bothrops asper utilizando extractos de dos plantas del género Phenax. Tesis de Licenciatura en Farmacia, Universidad de Costa Rica.

94.Cortés P., T.E. 1944. Diferentes especies del género Psidium, conocidas con el nombre de güísaro. Tesis de Licenciatura en Farmacia, Universidad de Costa Rica.

95.Coto Campos, J.M. 1978. Obtención de aminoácidos de la pulpa del café. Tesis de Licenciatura en Química, Universidad de Costa Rica.

96.Coto Rojas, R.A. 1988. Obtención de jarabe de glucosa de conversión intermedia por el método enzima-enzima a partir del banano verde. Tesis de Licenciatura en Ingeniería Química, Universidad de Costa Rica.

97.Cucurny, D. 1996. 101 traditional herbal remedies from Costa Rica. San José, Lit. León.

98.Chacón Araya, A.R. 1994. Producción de fructosa vía enzimática. Tesis de Licenciatura en Ingeniería Química, Universidad de Costa Rica.

99.Chacón Carballo, M. 2000. Determinación de las propiedades inmunoestimulantes de la Echinacea purpurea en la producción de anticuerpos en ratones inmunizados con veneno de Bothrops asper y en la proliferación de linfocitos in vitro. Tesis de Licenciatura en Farmacia, Universidad de Costa Rica.

100.Chacón Delgado, R. 1955. Un estudio de la toxicidad del Solanum mammosum. Tesis de Licenciatura en Farmacia, Universidad de Costa Rica.

101. Chang, G. et al. 1984. Remedios caseros y comidas tradicionales afrolimonenses. Recopilación. Ministerio de Cultura, Juventud y Deportes, Dep. Antropología. San José, Ministerio de Educación Pública.

102.Chavarría Poll, O. 1946. Constatación de los efectos piojicidas de las semillas de espuela de caballero, Delphinium ajacis. Tesis de Licenciatura en Farmacia, Universidad de Costa Rica.

103.Chaverri Chaverri, C.G. 1979. Estudio fitoquímico de las semillas y frutos de la especie Tabernaemontana arborea. Tesis de Licenciatura en Química, Universidad de Costa Rica.

104.Chaverri, C., Cicciò, J. \& Castro, V. 1983. Terpenoides de Montanoa dumicola Klatt. Ing. Cie. Quím. 7 (1-2): 13-14.

105. Chaverri, C., Cicciò, J.F. \& Calzada, J. 1984. Estudio fitoquímico de las hojas de Gyminda costaricensis Standl. (Celastraceae). Ing. Cie. Quím. 8(3-4): 52. 
106.Chaverri, C. \& Cicciò, J.F. 1980. Estudio preliminar de los frutos de Tabernaemontana arborea. Ing. Cie. Quím. 4(4): 152- 153.

107.Chaves Alfaro, P.R. 1994. Obtención de jarabe de glucosa de alta conversión vía método enzima-enzima a partir de banano verde. Tesis de Licenciatura en Ingeniería Química, Universidad de Costa Rica.

108.Cháves Gutiérrez, M.O. 1958. Ensayo bromatológico del Ziziphus mauritiana. Tesis de Licenciatura en Farmacia, Universidad de Costa Rica.

109. Chaves Solano, Y. 1994. Clasificación de aceites comerciales comestibles utilizando espectroscopía ultravioleta derivativa y métodos multivariados. Tesis de Licenciatura en Química, Universidad de Costa Rica.

110.Chaves, I.P., Cicciò, J.F. \& Poveda, L. 1980. Nuevos aceites de semillas de cuatro plantas tropicales. Ing. Cie. Quím. 4(4): 141.

111.Chaves, R. A. 1950. La guanábana, Annona muricata L. Análisis químico y estudio sobre su posible explotación. Tesis de Licenciatura en Farmacia, Universidad de Costa Rica.

112.Damián Cerdas, O. 2000. Formulación de un cosmético con actividad antiinflamatoria con extractos de las plantas Chaptalia nutans, Hamelia patens y Plantago major. Tesis de Licenciatura en Farmacia, UNIBE.

113.De la Cruz, R. 1961. Contribución al estudio químico de la Rauwolfia sarapiquensis. Tesis de Licenciatura en Farmacia, Universidad de Costa Rica.

114.Del Valle Leandro, J.E. 1963. Ensayo fitoquímico de la Trichilia havanensis, uruca, en Costa Rica. Tesis de Licenciatura en Farmacia, Universidad de Costa Rica.

115.Eisner, T. \& Niemeyer, H. 1996. Fármacos naturales. Rev. Divulg. Cient. Tecnol. Asoc. Ciencia Hoy 6(33).

116.Elizondo González, C. 1962. Estudio químico bromatológico de las hojas comestibles de Jatropha aconitifolia (chicasquil). Tesis de Licenciatura en Farmacia, Universidad de Costa Rica.

117.Espinoza Marín, C. 1996. Las maravillas del ajo, la cebolla y el limón. Colección Naturaleza y Salud (t.3). San José.

118.Esquivel B., L.M. 1963. Acción hipoglicemiante de la Bauhinia. Tesis de Licenciatura en Farmacia, Universidad de Costa Rica.

119.Esquivel Herrera, J. 1960. El tomillo cultivado en Costa Rica y su aceite esencial. Tesis de
Licenciatura en Farmacia, Universidad de Costa Rica.

120.Esquivel Rivera, O.M. 1997. Verificación de la actividad diurética de Cymbopogon citratus (DC.) Stapf, y evaluación de la toxicidad aguda de las plantas de Satureja viminea L. y Cymbopogon citratus. Tesis de Licenciatura en Farmacia, Universidad de Costa Rica.

121.Faingezicht Gutman, H. 1965. Algunas posibles utilizaciones del chan (Hyptis suaveolens) y de la linaza (Linum usitatissimum) en la industria farmacéutica. Tesis de Licenciatura en Farmacia, Universidad de Costa Rica.

122.Fallas Meléndez, P. 1994. Investigación de la presencia de alcaloides derivados del ácido lisérgico en semillas de la planta Ipomoea purpurea recolectadas en diferentes puntos del Valle Central. Importancia químico-forense. Tesis de Licenciatura en Química, Universidad de Costa Rica.

123. Ferraro Saborío, C. 1995. Método de bioautografía de capa de agar para la detección de actividad antifúngica en extractos de especies vegetales. Tesis de Licenciatura en Química, Universidad de Costa Rica.

124.Fresno, M. 1998. Hierbas medicinales y Santa Ana. Televisora de Costa Rica. Lo nuestro. (Videocasete)

125.Fuentes Sanabria, J.C. 1992. Estudio fitoquímico de la planta Croton hirtus. Tesis de Licenciatura en Química, Universidad de Costa Rica.

126.Gambassi Muñoz, E. 1971. Mecanismo de acción antiespasmódica intestinal de las flores de la Matricaria chamomilla L. Tesis de Licenciatura en Farmacia, Universidad de Costa Rica.

127. Gamboa, A. 1989. Efecto de un extracto liofilizado de Solanum melongena sobre la colesterolemia de ratas albinas. Tesis de Maestría en Ciencias Médicas, Universidad de Costa Rica.

128.Gamboa, W., Philipp, D. \& Serrano, O. 1996. Jardín botánico de plantas medicinales, aromáticas y plaguicidas del Valle de Ujarrás, Costa Rica. Ceiba 37(2): 317-321.

129.García González, M. 1984. Estudio preliminar de las acciones cardiovasculares de los extractos acuosos de las hojas de Casimiroa edulis (Llave et Lex.) en ratas albinas. Tesis de Licenciatura en Biología, Universidad de Costa Rica.

130.García González, M.1990. Efectos cardiovasculares del extracto acuoso y dos fracciones de Clusia coclensis D'Arcy administrado en ratas albinas normotensas e hipertensas. Tesis de Maestría en Ciencias Biomédicas, Universidad de Costa Rica. 
131.García González, M. 2000. Legislación en Iberoamérica sobre fitofármacos y productos naturales. San José, Editor Armando Cáceres, CYTED.

132.García González, M. \& Morales, O. 1996. Efectos cardiovasculares del extracto acuoso de las hojas de Clusia coclensis (Guttiferae). Rev. Biol. Trop. 44(1): 87-91.

133.García González, M. \& Morales, O. 1997. Efecto del extracto acuoso de las hojas de copey, Clusia coclensis (Clusiaceae), sobre la respuesta del reflejo barorreceptor en ratas. Rev. Biol. Trop. 45(3): 999-1003.

134.García González, M, González, C.S. \& Pazos, S.L. 1996-1997. Actividad farmacológica del extracto acuoso de la madera de Quassia amara (Simaroubaceae) en ratas y ratones albinos. Rev. Biol. Trop. 44 (3-4): 47-50.

135.García González, M. \& Morales, O. 1998. Acción vaso-periférica del extracto acuoso de las hojas de Clusia coclensis (Clusiaceae). Rev. Biol. Trop. 46(3): 575-578.

136.García González, M., Freer, B.E. \& Morales, O. 1994. Acciones de Casimiroa edulis (Rutaceae) sobre la presión arterial media y frecuencia cardíaca en ratas albinas. Rev. Biol. Trop. 42(1-2): 115-119.

137.García Segura, A. 1994. Plantas de la medicina bribrí. San José, Edit. Universidad de Costa Rica. $90 \mathrm{p}$.

138.Garnier, O. \& Nórval, H. 1956. Contribución al estudio de la raicilla de Costa Rica. Tesis de Licenciatura en Farmacia, Universidad de Costa Rica.

139.Ginzbarg, S. 1976. Medicinal plants used by the Bribri and Cabécar Indians of Costa Rica. Grinnell, Grinnell College, ACM Field Program.

140.Ginzbarg, S. 1977. Plantas medicinales de los indios bribrís y cabécar. Amér. Indíg. 37(2): $367-$ 398.

141.Gólcher Castro, I. 1962. Determinación de alcaloides en siete especies de la flora de Costa Rica. Tesis de Licenciatura en Farmacia, Universidad de Costa Rica.

142.Gómez Acosta, N. 1964. Análisis fitoquímico y farmacológico de la capitana (Verbesina tonduzii). Tesis de Licenciatura en Farmacia, Universidad de Costa Rica.

143.Gómez Laurito, J., Robles Valle, G. \& Villalobos Soto, R. 1998. Especies del género Smilax en Costa Rica. Plantas medicinales del género Smilax en Centroamérica. Reuniones Técnicas, 25 sept.
1997. Actas de la reunión. CYTED, RIPROFITO. Serie Técnica (CATIE) No. 2, p. 31-33. Turrialba.

144.Gómez, Cubillo, Mora \& Hilje. 1997. Evaluación de posibles repelentes de Bemisia tabaci. I. Productos comerciales. Man. Integr. Plagas 46: 9. 145.Gómez, Cubillo, Mora \& Hilje. 1997. Evaluación de posibles repelentes de Bemisia tabaci II. Extractos vegetales. Man. Integr. Plagas 46: 17.

146.González Castillo, E. 1959. Extracción del principio anestésico del alcotán. Tesis de Licenciatura en Farmacia, Universidad de Costa Rica.

147.González Guier, L. 1950. Estudio sobre la obtención, rendimiento y características del aceite esencial de orégano y del aceite esencial de agua florida en nuestro país. Tesis de Licenciatura en Farmacia, Universidad de Costa Rica.

148.González M., M. 1954. El poró; propiedades alimenticias de sus flores. Tesis de Licenciatura en Farmacia, Universidad de Costa Rica.

149.González Miranda, L. 1990. Mecanismo de la acción protectora antilitiásica renal de Senna alata L. Tesis de Licenciatura en Química, Universidad de Costa Rica.

150.Guido Gámez, T. 1951. Terminalia catappa (almendra tropical, almendra india). Análisis químico del fruto y de la nuez. Tesis de Licenciatura en Farmacia, Universidad de Costa Rica.

151.Guier C., M.A. 1942. Azul de mata, Jacobinia tinctoria: un estudio sobre su pigmento. Tesis de Licenciatura en Farmacia, Universidad de Costa Rica.

152.Gutiérrez Varela, G. 1951. Alcornoque (roble blanco), Licania arborea. Estudio del aceite encontrado en las semillas de este árbol, vulgarmente llamado alcornoque o roble blanco. Tesis de Licenciatura en Farmacia, Universidad de Costa Rica.

153.Guzmán, G. 1952. Ensayos con hojas de papaya en el tratamiento del reumatismo. Tesis de Licenciatura en Farmacia, Universidad de Costa Rica.

154.Guzmán Rodríguez, T. 1970. Efecto de la Matricaria chamomilla (manzanilla) sobre la musculatura lisa de yeyuno aislado. Tesis de Licenciatura en Farmacia, Universidad de Costa Rica.

155.Hammond, E.G., Wenchi, P.P. \& Mora-Urpí, J. 1982. Fatty acid composition and glyceride structure of the mesocarp and kernel oils of the pejibaye palm (Bactris gasipaes H.B.K.). Rev. Biol. Trop. 30(1): 91-93. 
156.Hasbun, C, Calderón, M. \& Castro, O. 1991 Nectandra membranacea. Alcaloides en su corteza y madera. Ing. Cie. Quím. 13(2): 19.

157.Hasbun, C., Calvo, M.A., Barrios, M., Arguedas, E., Calvo, A., Jiménez, R. \& Poveda, L. 1985. Distribución de friedelina en especies del género Clusia (Guttiferae) de Costa Rica Ing. Cie. Quím. 9(3): 96-97.

158.Hernández C, C. 1954. Estudio sobre el valor alimenticio de lo que en Costa Rica llamamos palo de papa (Calatola costaricensis) Standley. Tesis de Licenciatura en Farmacia, Universidad de Costa Rica.

159.Hernández Villalobos, J.H. 1959. Contribución al estudio de saponinas del itabo (Yucca elephantipes). Tesis de Licenciatura en Farmacia, Universidad de Costa Rica.

160.Hernández Villalobos, M. 1961. Estudio de las constantes físico-químicas del aceite de semilla de algodón (Gossypium hirsutum) producido en Costa Rica y de sus ácidos grasos. Tesis de Licenciatura en Farmacia, Universidad de Costa Rica.

161.Hidalgo Murillo, E. 1952. Estudio estadístico y comentarios farmacológicos sobre recetas formuladas en el año 1949. Tesis de Licenciatura en Farmacia, Universidad de Costa Rica.

162.Hirsch K., J. 1968. Determinación de berberina en el cardosanto de flores amarillas. Tesis de Licenciatura en Farmacia, Universidad de Costa Rica.

163.Hong On, N., Marín González, N. \& Zúñiga Gómez, L. 1977. Análisis alcaloidal preliminar de Magnolia poasana (Magnoliaceae). Tesis de Licenciatura en Farmacia, Universidad de Costa Rica.

164.Ingianna A., J. \& Gambassi M., E. 1982. Mecanismo de la acción antiespasmódica intestinal de las flores de Matricaria chamomilla L. Rev. Biol. Trop. 30(1): 85-90.

165. Ingianna Acuña, P. 1959. Investigación cuantitativa de oxalatos en algunas especies de nuestra flora y su poder lisante sobre los glóbulos rojos. Tesis de Licenciatura en Farmacia, Universidad de Costa Rica.

166.Inostrosa S., I. \& Fournier, L. 1982. Efecto alelopático de Gliricidia sepium (Jacq.) Steud. (madero negro). Rev. Biol. Trop. 30(1): 35-39.

167.Jiménez Bolaños, A.G. 1994. Extracción de rotenona a partir de las semillas de Pachyrhizus erosus (jícama). Tesis de Licenciatura en Ingeniería Química, Universidad de Costa Rica.

168.Jiménez Castro, S. \& Rodríguez Rodríguez, G.
2000. Inhibición de la actividad hemorrágica y proteolítica del veneno de Bothrops asper por extractos de las plantas Buddleja americana, Cissampelos pareira, Echinacea purpurea, Mikania guaco, Piper darienense y Vernonia patens. Tesis de Licenciatura en Farmacia, Universidad de Costa Rica.

169.Jiménez M., F. 1985. Evaluación del efecto hipotensor de algunas especies del género Eugenia en Costa Rica. Tesis de Licenciatura en Biología, Universidad Nacional, Heredia, Costa Rica.

170.Jiménez Monge, H. 1950. Aceites esenciales de ruda (Ruta chalepensis), romero (Rosmarinus officinalis) y tomillo (Thymus vulgaris). $\mathrm{Su}$ obtención a partir de plantas cultivadas en Costa Rica. Estudio de sus características. Tesis de Licenciatura en Farmacia, Universidad de Costa Rica.

171.Knobbs, E. 1996. Use and education of medicinal plants in the community of La Esperanza, Limón. San José. 62 p.

172.Koslowski, R. 1946. Rendimiento y calidad del aceite de cayeput obtenido del Melaleuca leucadendron Linn., cultivado en Costa Rica. Tesis de licenciatura en Farmacia, Universidad de Costa Rica.

173.León V., Y. 1958. Estudio del aceite esencial de la cáscara del banano (Musa sapientum L.). Tesis de Licenciatura en Farmacia, Universidad de Costa Rica.

174.León Varela, M. 1958. Estudio del aceite de la cáscara del limón dulce (Citrus limetta Risso). Tesis de Licenciatura en Farmacia, Universidad de Costa Rica.

175.León, S. \& Sanabria, F. 1977. Extracción de la lignina del bagazo y análisis de su estructura bioquímica. Ing. Cie. Quím. 1(2): 35-41.

176. Ling Nieto, F., Robles Valle, G. \& Villalobos Soto, R. 1998. Estudios ecológicos sobre zarzaparrilla y cuculmeca en Talamanca, Costa Rica. Plantas medicinales del género Smilax en Centroamérica. Reuniones Técnicas, 22-25 sept. 1997. Actas de la reunión. CYTED, RIPROFITO. Serie Técnica (CATIE) No. 2. Turrialba. p. 81-86.

177.Lizano R., R. 1964. Ensayos fitoquímicos y farmacológicos de la planta Gliricidia sepium Steudel, conocida en Costa Rica como madero negro. Tesis de Licenciatura en Farmacia, Universidad de Costa Rica.

178.Lobet L., U. 1954. El cardosanto amarillo. Tesis de Licenciatura en Farmacia, Universidad de Costa Rica. 
179.Lobo Gamboa, F. 1958. Estudio sobre el aceite de la semilla del zapote colorado (Calocarpum mammosum). Tesis de Licenciatura en Farmacia, Universidad de Costa Rica.

180.López Braga, R. 1988. Infusiones; buenas perspectivas de exportación. Tribuna Económ. 6(5): 32-33.

181.López V., J.A. \& Hernández M., E. 1981. Aislamiento de ácido cinámico y sacarosa en los frutos de Cassia grandis L. (Leguminosae). Ing. Cie. Quím. 5(2): 66.

182.López van der Laat, A. 1973. Efecto abortivo de las hojas de cafeto (Coffea arabica). Tesis de Licenciatura en Farmacia, Universidad de Costa Rica.

183.López, Barillas, Gómez-Laurito, Tyan, Al-Rehaily, Martin, Sharaf \& Schiff. 1997. Granulosin, a new chromone from Galipea granulosa. J. Natur. Prod. 60: 24 .

184.López, Barillas, Gómez-Laurito, Tyan, AlRehaily, Martin, Sharaf \& Schiff. 1997. Aporphine alkaloids of selected species of Nectandra and Ocotea (Lauraceae). Planta Med. 61: 493.

185.López, J.A. 1981. Aislamiento de cumarina en Zornia diphylla L. Ing. Cie. Quím. 5(3): 96.

186.López, J.A. 1982. Estudio fitoquímico de las semillas de Cordia collococca L. v. micrantha Swartz (Boraginaceae). Ing. Cie. Quím. 6(2): 157.

187.López, J.A. 1983. Aislamiento de acetato de Bamirina de hojas y tallos de caimito (Chrysophyllum cainito L.), Sapotaceae. Ing. Cie. Quím. 7 (1-2): 22-23.

188.López, J.A., Gómez Laurito, J., Lin, F., Duah, F.K., Sharaf, M., Aly, Y., Wong, L.K. \& Schiff, J.P. 1990. Alkaloids of Siparuna tonduziana. Planta Med. 56: 492.

189.López, J.A., Youssef, A. \& Schiff, J.P. 1988. Alkaloids of Siparuna pauciflora. Planta Med. 54(6): 552-553.

190.López, J.A. 1980. Aislamiento de escopoletina en Markea megalandra D'Arcy (M. leucantha Donn. Sm.). Ing. Cie. Quím. 4(4): 154.

191.López, J.A. 1981. Flavonoides de Cochlospermum vitifolium Willd. (Cochlospermaceae). Ing. Cie. Quím. 5(3): 101-102.

192.López, J.A. 1982. Aislamiento de quercitrina en las hojas de Anacardium rhinocarpus (A. excelsum). Ing. Cie. Quím. 6(1): 148-149.

193.López, J.A., Gómez Laurito, J., Lin, F., Sharaf, M., Wong, K. \& Schiff, J.P. 1993. Alkaloids of Siparuna griseo-flavescens. Planta Med. 59(2): 100.
194.López, J.A., Gómez Laurito, J., Lin, F., Sharaf, M., Wong, L.K. \& Schiff, J.P. 1993. Alkaloids of Guatteria diospyroides. Planta Med. 59(2): 191.

195.Luthmer Sánchez, C. 1947. Algunos ensayos con el látex del targuá (Croton gossypiifolius). Tesis de Licenciatura en Farmacia, Universidad de Costa Rica.

196.Madrigal Blanco, O. 1957. Ensayos sobre la acción farmacológica de la Verbesina tonduzii. Tesis de Licenciatura en Farmacia, Universidad de Costa Rica.

197.Madriz Masís, J.P. 1998. Usos potenciales de productos no maderables (PNM) del bosque tropical montano de la sección noroccidental de la Cordillera de Talamanca, Costa Rica. Informe de práctica de especialidad. Tesis de Bachiller en Ingeniería Forestal, Escuela de Ingeniería Forestal, Instituto Tecnológico de Costa Rica.

198.Madriz Masís, J.P. 1999. Exploración etnobotánica en los bosques húmedos tropicales de la Reserva Indígena Tayní, Costa Rica. Rev. For. Centroamer. (CATIE) 28: 22-26.

199.Madriz, J.P. 1997. Exploración etnobotánica de la flora silvestre comestible en los bosques húmedos tropicales de la reserva aborigen Tayní, Limón. III Congreso Forestal Nacional, 27-29 agosto. San José.

200.Maffioli Reyes, A. \& Ocampo S., R.A. 1987. El uso de algunas plantas medicinales en Costa Rica. $2^{\mathrm{a}}$. ed. San José, Litogr. e Impr. Lil.

201.Malavassi, E. 1942. Valor alimenticio del guineo. Tesis de Licenciatura en Farmacia, Universidad de Costa Rica.

202.Mancebo, Hilje, Mora \& Salazar. 2000. Antifeedant activity of Quassia amara (Simaroubaceae) extracts on Hypsipyla grandella (Lepidoptera: Pyralidae). Crop. Protec. 19: 301.

203.Marmillod, D., Chang, Y. \& Bedoya, R. 1997. Desarrollo de un plan de manejo para Quassia amara, un recurso no maderable del bosque tropical. CATIE, Programa de Investigación. 3. Semana Científica. 3-5 Feb 1997. Impr. Turrialba, Costa Rica. p. 23-28.

204.Marquina, M. \& Trabanino, E. 1994. Medicina natural: una alternativa para la salud. Hombres de Maíz 27: 40-41.

205.Martínez U., P. 1963. Ensayo bromatológico del Solanum topiro H.B.K. Tesis de Licenciatura en Farmacia, Universidad de Costa Rica.

206. Martínez Urtubia, N. 1960. Estudio comparativo de la actividad proteolítica de las papaínas obtenidas de la Carica peltata Hook. et Arn. 
y la Carica papaya L. Tesis de Licenciatura en Farmacia, Universidad de Costa Rica.

207.Mata Arias, C. 1944. Algunas consideraciones y ensayos sobre la producción comercial de drogas botánicas en Costa Rica. Rev. Univ. Costa Rica 1: 4-17, 72-77.

208. Mata Sandí, M.V. 1994. Nuevo estudio fitoquímico de la planta Lycoseris latifolia D. Don. Tesis de Licenciatura en Química, Universidad de Costa Rica.

209.Matamoros Murillo, E.M. 1962. Porcentaje de vitamina $\mathrm{C}$ y alcaloides de naranjilla. Tesis de Licenciatura en Farmacia, Universidad de Costa Rica.

210.Maug Acuña, M.V. 1994. Efecto del escaldado sobre el secado y la calidad físico-química de la pimienta negra (Piper nigrum L.). Tesis de Licenciatura en Tecnología de Alimentos, Universidad de Costa Rica.

211.Mazzali B., G. 1955. Colaboración al estudio de la teobromina en cacao nacional. Tesis de Licenciatura en Farmacia, Universidad de Costa Rica.

212. Mejía Ramírez, J. 1992. Algunas plantas sanativas de Costa Rica. $1^{\text {a }}$. ed. San José, Costa Rica.

213. Méndez Arrieta, Y. 1958. Contribución al estudio de las saponinas y sapotoxinas presentes en algunas plantas de nuestra flora. Tesis de Licenciatura en Farmacia, Universidad de Costa Rica.

214.Ministerio de Educación Pública (MEP). 1986. Primer recetario de medicina vegetal: plantas curativas y tratamientos homeopáticos. Dirección Regional de Enseñanza. Región Central. San José, Costa Rica.

215.Miranda Arrieta, T. \& Vargas Vargas, K. 1996. Búsqueda de la actividad gastrointestinal del extracto acuoso bruto de Quassia amara L. (Simaroubaceae). Tesis de Licenciatura en Farmacia, Universidad de Costa Rica.

216. Miranda M., M. 1952. Ensayos con el saragundí en el tratamiento del reumatismo. Tesis de Licenciatura en Farmacia, Universidad de Costa Rica.

217.Miranda Quesada, A. \& Campos Montero, V.M. 1966. Separación cromatográfica del principio activo de la especie Pernettya coriacea Klot. Tesis de Licenciatura en Farmacia [de A.M.Q.], Universidad de Costa Rica.

218.Mojica Araya, E. 1983. Estudio fitoquímico de la raíz de la planta Gyminda costaricensis Standl. (Celastraceae). Tesis de Licenciatura en Química, Universidad de Costa Rica.
219.Monge Valverde, R. 1956. Estudio comparativo de los aceites esenciales del culantro de Castilla (Coriandrum sativum) y culantro de coyote (Eryngium foetidum) cultivados en Costa Rica. Tesis de Licenciatura en Farmacia, Universidad de Costa Rica.

220.Montealegre, L., Portugués, P., Chaverri, A., Vega, F. \& Calzada, J. 1987. Industrialización del tamarindo (Tamarindus indica) en Costa Rica. Ing. Cie. Quím. 11(1): 35-37.

221. Montes de Oca, F. 1955. Ensayo del guijarro como raticida. Tesis de Licenciatura en Farmacia, Universidad de Costa Rica.

222.Montiel Calderón, H. 1997. Desarrollo de una técnica no destructiva para la determinación del producto cosechable de plantas medicinales de Smilax chiriquensis C.V. Morton (Smilacaceae) en la Reserva Indígena Kéköldi, Baja Talamanca, Limón. Informe de Práctica de Especialidad. Escuela de Ingeniería Forestal, Instituto Tecnológico de Costa Rica.

223.Montiel, H., Villalobos, R., Marmillod, D., Ocampo, R. \& Valerio, J. 1997. Identificación de herramientas para la estimación de existencias de Smilax chiriquensis (recurso no maderable medicinal) en bosques naturales. 3. Congreso Forestal Centroamericano, 15-17 sept., San José, Impresos Belén. p. 37-39.

224.Mora Bolaños, W.D. 1994. Estudio fitoquímico de la planta Ageratina cartagoensis K. \& R. Tesis de Licenciatura en Química, Universidad de Costa Rica.

225. Mora Fonseca, E. 1957. Aceite esencial de anisillo. Tesis de Licenciatura en Farmacia, Universidad de Costa Rica.

226.Morales Mora, M.A. 1962. Contribución al estudio de la Averrhoa carambola L., carambola. Tesis de Licenciatura en Farmacia, Universidad de Costa Rica.

227.Morales Sánchez, G. 1976. Determinación de lobelina en Lobelia laxiflora HBK. Tesis de Licenciatura en Farmacia, Universidad de Costa Rica.

228.Morales Ujueta, W. 1971. Nombre común, científico y usos de algunas plantas medicinales costarricenses. San José.

229.Morales Ujueta, W. 1973. Algunas plantas medicinales costarricenses de uso popular en el Valle Central. Facultad de Farmacia, Universidad de Costa Rica.

230.Morales Vargas, M.H. 1971. Alcaloides indólicos en Ipomoea parasitica. Tesis de Licenciatura en 
Farmacia, Universidad de Costa Rica.

231.Moreno, A.H. 1985. Sistemas agroforestales con Gliricidia sepium. In: Beer, J.W., Fassbender, H.W. \& Heuveldop, J. (eds.). Seminario Avances en la Investigación Agroforestal. 1-11 sept., CATIE, Turrialba. Deutsche Gesellschaft für Technische Zusammenarbeit (GTZ); Proyecto Agroforestal CATIE/GTZ. Serie Técnica. Informe Técnico (CATIE) No. 147. p. 214-221.

232.Morera, J.A. (ed.). 1983. Seminario sobre la problemática del cultivo de las plantas medicinales y especias en Costa Rica. 9-10 jun., CATIE, Unidad de Recursos Fitogenéticos. Turrialba.

233. Morux Araya, S. 1964. Análisis fotoquímico de la gavilana (Neurolaena lobata). Tesis de Licenciatura en Farmacia, Universidad de Costa Rica.

234.Mundina, M., Vila, R., Tomi, F., Cicciò, J.F., Ibáñez, C., Adzet, T., Casanova, J., Cañigueral, S. 2000. Composition of the essential oils from leaves and fruits of three Hedyosmum species from Costa Rica. Flavour Fragr. J. 15: 201-205.

235.Muñoz C., L., Castro, O., López, C.R., Arias, A.R., Pignani, F. \& Calzada, J. 1982. Potencial nematicida en nuevas fuentes naturales de plantas pertenecientes al género Tagetes (familia Compositae). Ing. Cie. Quím. 6(1): 158-160.

236. Murillo Alfaro, L. 1960. Estudio del aceite esencial de las raíces y de las flores del Hedychium coronarium Koenig, conocido en Costa Rica como heliotropo blanco. Tesis de Licenciatura en Farmacia, Universidad de Costa Rica.

237.Murillo Masís, R.M. 1992. Estudio fitoquímico preliminar de Croton xalapensis Kunth. Tesis de Licenciatura en Química, Universidad de Costa Rica.

238.Murillo, R. \& Jakupovic, J. 1998. Glicósidos de Euphorbia aucherii. Ing. Cie. Quím. 18 (2): 57.

239. Murillo, R. \& Jakupovic, J. 2000. Clerodanos y secoclerodanos de Croton jimenezii (Euphorbiaceae). Ing. Cie. Quím. 19(2): 68-73.

240.Murillo, R. \& Castro, V.H. 1998. Un alcaloide del tipo harmano aislado de la Psychotria suerrensis (Rubiaceae). Ing. Cie. Quím. 18: 61.

241. Museo Nacional de Costa Rica. 1989. Resumen del I Seminario Mesoamericano de Etnofarmacología y II Congreso Nacional de Medicina. San José.

242.Nader, W., Rojas, M., Robles, G. \& Villalobos, R. 1998. Plantas medicinales del género Smilax en Centroamérica. Reuniones Técnicas, 22-25 sept. 1997. Turrialba. Actas de la reunión. CYTED; RIPROFITO. Serie Técnica (CATIE) No. 2, p. 23-27.
243.Nanne E., C.I. \& Aragón, O. 1991. Aislamiento, purificación y caracterización de una lectina de la semilla del poró, Erythrina costaricensis (Leguminosae). Rev. Biol. Trop. 39: 15-21.

244.Nassar Carballo, M. 1961. Ensayo fitoquímico de la especie Thevetia plumeriaefolia, chirca venenosa, en Costa Rica. Tesis de Licenciatura en Farmacia, Universidad de Costa Rica.

245.Nassar Carballo, M., Sáenz R., J.A. \& Gálvez, N. 1980. Phytochemical screening of Costa Rican plants: Alkaloid analysis. V. Rev. Biol. Trop. 28(1): 1-11.

246.Navarro Mairena, C.E. 1976. Las principales plantas medicinales de Costa Rica. ITCO, Depto. de Organización y Capacitación Campesina, Serie Divulgación Campesina No. 8. 27 p. San José.

247.Nema F., C.M. 1947. Spigelia anthelmia (lombricera). Investigación del alcaloide espigelia en la planta costarricense. Tesis de Licenciatura en Farmacia, Universidad de Costa Rica.

248.Núñez Meléndez, E. 1982. Plantas medicinales de Costa Rica y su folclore. $3^{\mathrm{a}}$. ed. San José, Edit. Univ. Costa Rica.

249.Nygren, A. 1992. Traditional uses and cultural significance of three Erythrina species among the rural population of Tuís District, Turrialba, Costa Rica. In: Westley, S.B. \& Powell, M.H. (eds.). International Conference: Erythrina in the New and Old Worlds. CATIE, Turrialba, 19-23 oct. p. 62-67. Serie: Nitrogen Fixing Tree Research Reports (EUA). Special issue.

250.Ocampo Alvarado, M.F. 1957. La chirca, Thevetia peruviana. Los principios activos del fruto. Tesis de Licenciatura en Farmacia, Universidad de Costa Rica.

251.Ocampo S., R.A. 2000. Agrotecnología para el cultivo de ipecacuana o raicilla, Psychotria ipecacuanha (Brot.) Stokes. In: Martínez A., J.V., Yesid Bernal, H. \& Cáceres, A. (eds.). Fundamentos de agrotecnología de cultivo de plantas medicinales iberoamericanas. Programa Iberoamericano de Ciencia y Tecnología. Subprograma X. Química Fina Farmacéutica. Santa Fe de Bogotá, Colombia. Secretaría Ejecutiva del Convenio Andrés Bello. SECAB: Ciencia y Tecnología 83: 357-374.

252.Ocampo S., R.A. \& Robles, G., 2000. Agrotecnología para el cultivo de zarzaparrilla o saskecha, Smilax chiriquensis C. Morton. In: Martínez A., J.V., Yesid Bernal, H. \& Cáceres, A. (eds.). Fundamentos de agrotecnología de cultivo de plantas medicinales iberoamericanas. Programa Iberoamericano de Ciencia y Tecnología. 
Subprograma X. Química Fina Farmacéutica. Santa Fe de Bogotá, Colombia. Secretaría Ejecutiva del Convenio Andrés Bello. SECAB: Ciencia y Tecnología 83: 421-432.

253.Ocampo S., R.A. \& Villalobos Soto, R. 2000. Agrotecnología para el cultivo de hombre grande o cuasia, Quassia amara Linn. In: Martínez A., J.V., Yesid Bernal, H. \& Cáceres, A. (eds.). Fundamentos de agrotecnología de cultivo de plantas medicinales iberoamericanas. Programa Iberoamericano de Ciencia y Tecnología. Subprograma X. Química Fina Farmacéutica. Santa Fe de Bogotá, Colombia. Secretaría Ejecutiva del Convenio Andrés Bello. SECAB: Ciencia y Tecnología 83: 375-386.

254.Ocampo S., R.A. \& Maffioli, A. 1985. El uso de algunas plantas medicinales en Costa Rica. San José, Impr. Trejos.

255.Ocampo S., R.A. \& Villalobos Soto, R. 1995. El hombre grande, planta insecticida y medicinal. Guía Agropec. Costa Rica 12(26): 20-21.

256.Ocampo S., R.A. \& Villalobos Soto, R. 1996. Experiencias técnicas sobre domesticación de plantas medicinales en Centroamérica. Seminario sobre Industrialización y Legalización de Productos Fitofarmacéuticos en Iberoamérica; Reunión Constitutiva de la Red Iberoamericana de Productos Fitofarmacéuticos (RIPROFITO). Antigua Guatemala, 28 sept.-1 oct. Programa Iberoamericano de Ciencia y Tecnología para el Desarrollo. Subprograma X. Química Fina Farmacéutica. p. 60-65.

257.Ocampo S., R.A. (ed.). 1995. Potencial de Quassia amara como insecticida natural. Reunión Centroamericana. Informe Técnico (CATIE) No. 267. Turrialba.

258.Ocampo S., R.A. 1983. Algunas plantas medicinales de la región atlántica. San José, Instituto de Desarrollo Agrario (IDA). 42 p.

259.Ocampo S., R.A. 1986. Jardines para la salud. Serie informática; Tecnología Apropiada No. 11. Centro de Información Tecnológica, Instituto Tecnológico de Costa Rica.

260.Ocampo S., R.A. 1992. Seminario sobre el cultivo de especias en Costa Rica. Programa Cooperativo Universidad de Costa Rica - IDA; Colegio de Ingenieros Agrónomos. Liberia, Universidad de Costa Rica.

261.Ocampo S., R.A. 1994. Domesticación de plantas medicinales en Centroamérica. In: Ocampo S., R.A. (ed). Reunión Técnica Centroamericana: Domesticación de Plantas Medicinales en Centroamérica. Turrialba. Informe Técnico
(CATIE) No. 245.

262.Ocampo S., R.A. 1997. Estado de avance en la agroindustria de plantas medicinales en Costa Rica. Agronomía Costarricense 21(1).

263.Ocampo S., R.A., Valverde Castro, R.U. \& Fuentes Fiallo, V.R. 2000. Manual de cultivo y conservación de plantas medicinales. San José, TRAMIL.

264.Ocampo Solano, M.A. 1951. Análisis de los ruibarbos que se encuentran en Costa Rica (Rumex crispus y Rumex obtusifolius) y comparación con los oficiales (Rheum officinale y Rheum palmatum). Tesis de Licenciatura en Farmacia, Universidad de Costa Rica.

265.Ocampo, R.A. 1996. Situación del estado de avance en la agroindustria de plantas medicinales en Costa Rica. In: Bertsch, F., Badilla, W. \& García, J. (eds.). Memoria: ¿Puede la agricultura sostenible ser competitiva? Agronomía y recursos naturales. 10. Congreso Nacional Agronómico y de Recursos Naturales; 3. Congreso Nacional de Fitopatología; 2. Congreso Nacional de Suelos. 8-12 jul., San José. Asociación Costarricense de la Ciencia del Suelo; Colegio de Ingenieros Agrónomos; Asociación Costarricense de Fitopatólogos. EUNED/EUNA. p. 11-15.

266.Ocampo, R.A. 1984. Las comunidades indígenas dentro del contexto del Parque de la Biosfera La Amistad. Congreso Mundial sobre Parques Culturales. Mesa Verde. Colorado (EE.UU.), 17-21 sept.

267.Ocampo, R.A. 1996. Situación de la agroindustria de productos naturales en Costa Rica. Seminario sobre Industrialización y Legalización de Productos Fitofarmacéuticos en Iberoamérica. Reunión Constitutiva de la Red Iberoamericana de Productos Fitofarmacéuticos (RIPROFITO). Antigua Guatemala, 28 sept.-1 oct. Programa Iberoamericano de Ciencia y Tecnología para el Desarrollo. Subprograma X. Química Fina Farmacéutica. p. 139-142.

268.Ocampo, S. \& Maffioli, A. 1987. El uso de algunas plantas medicinales en Costa Rica. Vol. I. $2^{\mathrm{a}}$. ed. San José, Imprenta Lil.

269.Oconitrillo Segura, J. 1998. Perfil de mercado de 10 plantas medicinales para la empresa agroindustrial Coopegarabito R.L. Informe de Práctica de Especialidad en Bachillerato. Escuela de Ingeniería Agropecuaria Administrativa, Instituto Tecnológico de Costa Rica. 118 p.

270.Ochoa López, V.L. 1997. Los conocimientos de la mujer y el hombre sobre el manejo de las plantas alimenticias y medicinales, dentro de los sistemas 
de producción campesina en fincas pequeñas / Woman and man knowledge about the use and management of nutritious and medicinal plants in the peasant production systems in small farms. Tesis de Maestría, CATIE, Turrialba.

271.Ochoa, L., Fassaert, C., Somarriba, E. \& Schlönvoigt, A. 1998. Conocimiento de mujeres $\mathrm{y}$ hombres sobre las especies de uso medicinal y alimenticio en huertos caseros de Nicoya, Costa Rica / Men and women's knowledge about medicinal and food species in home gardens of Nicoya, Costa Rica. Agrofor. Amér. (CATIE) 5(17-18): 7-11.

272.Ochoa, L., Fassaert, C., Somarriba, E. \& Schlonvoigt, A. 1999. Medicinal and food plants in Nicoya, Costa Rica: there are differences in what men know and what women know. Agrofor. Today (Kenia) 11(1-2): 11-12.

273. Oreamuno O., F. 1953. El raspaguacal (Crescentia cujete L.): Estudio sobre un remedio de uso popular. Tesis de Licenciatura en Farmacia, Universidad de Costa Rica.

274. Orlich R., B. 1949. Algunos datos sobre la Cucurbita (pipián). Tesis de Licenciatura en Farmacia, Universidad de Costa Rica.

275.Ortega Meza, M.A. 1963. Análisis bromatológico de la piñuela (Bromelia pinguin). Tesis de Licenciatura en Farmacia, Universidad de Costa Rica.

276. Ovares Jenkins, L. 1950. Bálsamo del Perú: su posible explotación en Costa Rica. Tesis de Licenciatura en Farmacia, Universidad de Costa Rica.

277.Palma Z., T., Gadea Baltodano, A. \& Chaves, A. 2000. El cultivo de la raicilla, Psychotria ipecacuanha. Colección Productos no Maderables del Bosque. Instituto Tecnológico de Costa Rica (ITCR).

278.Palma Zúñiga, T. 1999. Especias y plantas medicinales. Tecnol. en Marcha 13(1): 16-19.

279. Palma, T. 1996. Biotecnología en la domesticación de plantas medicinales. In: Bertsch, F., Badilla, W. \& García, J. (eds.). Memoria: ¿Puede la agricultura sostenible ser competitiva? Agronomía y recursos naturales. 10. Congreso Nacional Agronómico y de Recursos Naturales; 3. Congreso Nacional de Fitopatología; 2. Congreso Nacional de Suelos. San José, 8-12 jul. Asociación Costarricense de la Ciencia del Suelo; Colegio de Ingenieros Agrónomos; Asociación Costarricense de Fitopatólogos. EUNED/EUNA. p. 31-34.

280.Palma, T. 1996. Caracterización de los sectores asociados a la producción, comercialización y transformación de plantas medicinales en Costa Rica. In: Bertsch, F., Badilla, W. \& García, J. (eds.). Memoria: ¿Puede la agricultura sostenible ser competitiva? Agronomía y recursos naturales. 10. Congreso Nacional Agronómico y de Recursos Naturales; 3. Congreso Nacional de Fitopatología; 2. Congreso Nacional de Suelos. 8-12 jul., San José. Asociación Costarricense de la Ciencia del Suelo; Colegio de Ingenieros Agrónomos; Asociación Costarricense de Fitopatólogos. EUNED/EUNA. p. 23-29.

281.Palmer, P., Sánchez, J. \& Mayorga, G. 1991. Taking care of Sibö's gifts: an environmental treatise from Costa Rica's Kéköldi Indigenous Reserve. Asociación de Desarrollo Integral de la Reserva Indígena Cocles/Keköldi. San José. 96 p.

282.Peñalba Terreros, R. 1984. Efectos de extractos liofilizados de Momordica charantia sobre la glicemia y la presión arterial en preparaciones caninas agudas y crónicas. Tesis de Maestría en Ciencias Biomédicas, Universidad de Costa Rica.

283.Peralta, F. 1946. Sirrí (Tapirira brenesii Standl.). Estudio del aceite encontrado en las semillas de este árbol vulgarmente llamado sirrí o ciruelo del monte. Tesis de Licenciatura en Farmacia, Universidad de Costa Rica.

284.Pérez Castillo, A.G. 1993. Evaluación fitoquímica del follaje de Phoebe hammeliana. Tesis de Licenciatura en Química, Universidad de Costa Rica.

285.Pérez, E.M. 1947. Investigación en Derris sp. Tesis de Licenciatura en Farmacia, Universidad de Costa Rica.

286.Pérez, R. \& Ocampo S., R. 1995. Análisis sobre la producción y comercialización de plantas medicinales en tres comunidades del Caribe de Costa Rica. Documento de Trabajo (CATIE) No.15. Turrialba.

287.Peters Reinford, M. 1982. Obtención de alcohol a partir de yuca. Tesis de Licenciatura en Tecnología de Alimentos, Universidad de Costa Rica.

288.Pittier, H. 1978. Plantas usuales de Costa Rica. San José, Edit. Costa Rica.

289.Piza L., J. \& Koss, R.M. 1985. Estudio preliminar de la obtención y caracterización de pectinas a partir de residuos de naranjas de la variedad criolla del cantón de Acosta, San José. Ing. Cie. Quím. 9(2): 49-52.

290.Poveda A., L.J. 1984. Lo maravilloso de nuestra flora medicamentosa. Biocenosis 1 (1): 22-23. 
291.Prado Cubero, A. 1964. Análisis bromatológico de la cebada malteada y su posible uso como base en la fabricación de un forraje completo. Tesis de Licenciatura en Farmacia, Universidad de Costa Rica.

292.Procúpez Schtirbu, R. 1994. Estudio fitoquímico del tallo y de las hojas de la especie Myrrhidendron donnell-smithii. Tesis de Licenciatura en Química, Universidad de Costa Rica.

293.Quesada Heinze, H. 1951. Análisis comparativo entre el café (Coffea arabica L.) y el café ñanjú (Hibiscus esculentus). Tesis de Licenciatura en Farmacia, Universidad de Costa Rica.

294.Quesada V., F. 1954. El ojoche, Brosimum terrabanum Pittier. Estudio del fruto, un alimento de nuestras costas. Tesis de Licenciatura en Farmacia, Universidad de Costa Rica.

295.Quesada Vargas, R. 1966. Ensayos cromatográficos de extractos de algunas especies de las familias Apocynaceae y Solanaceae. Tesis de Licenciatura en Farmacia, Universidad de Costa Rica.

296.Quesada, C.E. 1954. Contribución al estudio del Solanum ciliatum (naranjilla de color). Tesis de Licenciatura en Farmacia, Universidad de Costa Rica.

297.Quirós Calvo, M. 1951. Botánica aplicada a la Farmacia. Escuela de Farmacia, Universidad de Costa Rica. Tomo 1. San José, Costa Rica.

298.Quirós Calvo, M. 1945-1951. Botánica aplicada a la farmacia; comprende las especies extranjeras y plantas de Costa Rica a las divisiones aquí estudiadas. San José, Edit. Universitaria.

299.Quirós, M. 1936. Contribución al estudio de las plantas medicinales de Costa Rica. Ciencia 1.

300.Ramírez Guevara, O. 1952. Estudio sobre el valor alimenticio de lo que en Costa Rica se llama rabo de mico. Tesis de Licenciatura en Farmacia, Universidad de Costa Rica.

301.Ramírez Matamoros, A. 2001. Estudio de la inhibición de la actividad mionecrótica e inflamatoria inducida por el veneno de Bothrops asper de extractos acuosos de las plantas Buddleja americana, Cissampelos pareira, Echinacea purpurea y Vernonia patens. Tesis de Licenciatura en Farmacia, Universidad de Costa Rica.

302.Ramírez Zeledón, V. 1950. Las mentas de Costa Rica (yerbabuena) y su rendimiento en aceite esencial. Tesis de Licenciatura en Farmacia, Universidad de Costa Rica.

303.Ramos Guerrero, R.A. 1962. Ensayo fitoquímico de la especie Cissampelos pareira (pareira brava). Tesis de Licenciatura en Farmacia, Universidad de
Costa Rica

304.Rastrelli, Mora, Poveda \& Aquino. 2001. Phenolic constituents of Phenax angustifolius. J. Natur. Prod. 64: 79.

305.Redondo G., J.F. 1940. Estudio analítico de la verdolaga. Tesis de Licenciatura en Farmacia, Universidad de Costa Rica.

306.Redondo Valle, M.A. 1959. Ensayo de la planta Hymenaea courbaril (guapinol) en el tratamiento de la diabetes mellitus. Tesis de Licenciatura en Farmacia, Universidad de Costa Rica.

307.Reinhard Carrillo, M. 1949. Estudio sobre el Pilocarpus racemosus Vahl (talcacao) de Costa Rica. Ensayo para constatar el porcentaje de alcaloides y su posible rendimiento económico. Tesis de Licenciatura en Farmacia, Universidad de Costa Rica.

308.Revelo S., M. 1946. Arracache (Arracacia atropurpurea). Algunos datos sobre su valor alimenticio. Tesis de Licenciatura en Farmacia, Universidad de Costa Rica.

309. Rivas Bonilla, G. 1949. Estudio sobre la obtención y rendimiento de algunos aceites esenciales en nuestro país. Tesis de Licenciatura en Farmacia, Universidad de Costa Rica.

310.Rivera Zúñiga, M.F. 1994. Estudio de prefactibilidad para una planta de extracción de emetina y cefelina partiendo de raicilla - ipecacuana - de San Carlos. Tesis de Licenciatura en Ingeniería Química, Universidad de Costa Rica.

311.Rodríguez Caldera, M. 1969. Contenido de compuestos antracénicos en varias especies de Cassia de la flora costarricense. Tesis de Licenciatura en Farmacia, Universidad de Costa Rica.

312.Rodríguez Castro, E. \& Rojas Carrera, F. 1995. Curación con plantas. San José, Costa Rica.

313.Rodríguez Matamoros, J. 1944. Investigación del contenido de vitamina $\mathrm{C}$ en algunas legumbres de Costa Rica. Tesis de Licenciatura en Farmacia, Universidad de Costa Rica.

314.Rodríguez Navas, H. 1989. Uso de las plantas medicinales como plaguicidas orgánicos. In: Bolaños Arquin, M. \& Bolaños Arquin, I. (eds.). Memoria Primer Simposio sobre Tecnología Apropiada y Agricultura Biológica para un Desarrollo Rural Alternativo. Turrialba, 26-28 jul. Universidad de Costa Rica, Coordinadora de Organismos no Gubernamentales con Proyectos Alternativos de Desarrollo. San José, Edit. Universidad de Costa Rica. p. 101-105.

315.Rodríguez Navas, H. 1996. El maravilloso mundo 
de las plantas medicinales. Alajuela, Edit. Alfalit.

316.Rodríguez Navas, H. 2000. La utilidad de las plantas medicinales en Costa Rica. EUNA, Heredia.

317.Rodríguez Ortiz, I. 1986. Estudio preliminar para la industrialización de la fruta de pan (Artocarpus altilis). Tesis de Licenciatura en Tecnología de Alimentos, Universidad de Costa Rica.

318.Rodríguez Rodríguez, G. 1988. Alcaloides en hojas de Nectandra membranacea. Tesis de Licenciatura en Química, Universidad de Costa Rica.

319.Rodríguez Rodríguez, G. 1990. Alcaloides en hojas de Nectandra membranacea. Cie. Tecnol. 14(1-2): 219.

320.Rodríguez S., O.R. 1960. Ensayo fitoquímico de la especie Hymenaea courbaril L. (guapinol) en Costa Rica. Tesis de Licenciatura en Farmacia, Universidad de Costa Rica.

321.Rodríguez Vargas, C. 1960. Investigación del principio urticante de algunas ortigas de Costa Rica. Tesis de Licenciatura en Farmacia, Universidad de Costa Rica.

322.Rojas A., A.L., Vásquez V., L.A., Angulo A., D., \& Hernández S., I. 1987. Las plantas medicinales en la comunidad de Palmares, Alajuela, Costa Rica.

323.Rojas Carrera, F. 1999. Plantas medicinales: tratamientos, enfermedades, patologías, sintomatologías. San José.

324.Rojas Prado, G. 1958. Algunos ensayos farmacológicos con la hoja del carao (Cassia grandis L.f.). Tesis de Licenciatura en Farmacia, Universidad de Costa Rica.

325.Rojas Rojas, A. 1988. Estudio fitoquímico de Ageratina ligustrina (Compositae). Tesis de Licenciatura en Química, Universidad de Costa Rica.

326.Rojas Rojas, A. 1990. Estudio fitoquímico de Ageratina ligustrina (Compositae). Cie. Tecnol. 14(1-2): 221-222.

327.Rojas, A., Castro, V., Cicciò, J.F. \& Tamayo, G. 1988. Estudio fitoquímico de Ageratina ligustrina (D.C.) King et Rob. (Compositae). Ing. Cie. Quím. 12(1-2): 10-11.

328. Romero Chacón, R.M. 1990. Estudio fitoquímico de Senecio costaricensis y Senecio oerstedianus. Tesis de Licenciatura en Química, Universidad de Costa Rica.

329. Romero Chacón, R. 1990. Estudio fitoquímico de Senecio costaricensis y Senecio oerstedianus. Cie. Tecnol. 14(1-2): 220.

330.Romero Chacón, R.M. 1994. Enzymes of chorismate metabolism in Ailanthus altissima (Mill.) Swingle cell cultures. London, Sydney.

331.Romero Jiménez, M. 1989. Acción cardiovascular de extractos acuosos de hojas de Syzygium jambos (L.) Alston. Tesis de Maestría en Ciencias Biomédicas, Universidad de Costa Rica.

332.Romero, R., Castro, V. \& Cicciò, J. 1990. Estudio fitoquímico de Senecio costaricensis R.M King (Compositae). Rev. Latinoamer. Quim. 21(2): 9091.

333.Romero, R., Castro, V. \& Cicciò, J.F. 1991. Furanoeremofilonas adicionales de Senecio oerstedianus Benth. ex Oerst. Ing. Cie. Quím. 13(3): 24.

334. Rowe Hamilton, N. 1967. Estudio farmacognósico cualitativo de la corteza de Spondias purpurea L. Tesis de Licenciatura en Farmacia, Universidad de Costa Rica.

335.Rueda Porras, M. 1949. Posibilidades del aprovechamiento de la jalapa (Exogonium jalapa) silvestre en Costa Rica. Tesis de Licenciatura en Farmacia, Universidad de Costa Rica.

336.Ruiz Flores, E.I. 1990. Estudio fitoquímico de las hojas de Ocotea holdridgeiana, familia Lauraceae. Tesis de Maestría en Química, Universidad de Costa Rica.

337.Rundo, M. E. 1994. Índice primero de conocimientos y aplicaciones del ajo, ajenjo, altamisa, anís, albahaca, bardana, berro, borraja. Hierbería Las Tres Américas, San José, Costa Rica.

338.Rüngeler, Castro, Mora, Heike \& Merfort. 1998. Study of three sesquiterpene lactones from Tithonia diversifolia (Asteraceae) on their anti-inflamatory activity using the transcription factor NF-kb and enzymes of the arachidonic acid pathways as targets. Planta Med. 64: 588.

339.Sáenz de Rodríguez, V. 1959. Estudio comparativo del contenido de aceites esenciales de dos especies de Piper. Tesis de Licenciatura en Farmacia, Universidad de Costa Rica.

340.Saénz Núñez, M. 1951. Análisis de cuatro variedades de frijoles nacionales. Tesis de Licenciatura en Farmacia, Universidad de Costa Rica.

341.Sáenz, J.A. 1964. Contribución al estudio fitoquímico de las plantas costarricenses. I. Análisis alcaloidal. Rev. Biol. Trop. 12(1): 67-74.

342.Sáenz, J.A. \& Nassar, M. 1961. Glicósidos de Thevetia plumeriaefolia Benth. I. Rev. Biol. Trop. 9(2): 233-239.

343.Sáenz, J.A. \& Nassar, M. 1968. Phytochemical 
screening of Costa Rican plants: Alkaloid Analysis. III. Rev. Biol. Trop. 15(1): 195-202.

344.Sáenz, J.A. \& Nassar, M. 1969. Estudio toxicológico y fitoquímico de Pernettya coriacea Klotzsch. Rev. Biol. Trop. 15(2): 249-257.

345.Sáenz, J.A. \& Nassar, M. 1971. Phytochemical screening of Costa Rican plants: Alkaloid Analysis. IV. Rev. Biol. Trop. 18(1-2): 129-138.

346.Sáenz, J.A. \& Nassar, M. 1972. Toxic effect of the fruit of Passiflora adenopoda D.C. on humans: phytochemical determination. Rev. Biol. Trop. 20(1):137-140

347.Sáenz, J.A. 1964. Melochia pyramidata L. I. Análisis alcaloidal y cromatográfico; informe preliminar. Rev. Biol. Trop. 12(2): 157-163.

348.Sáenz, J.A. 1965. Phytochemical screening of Costa Rican plants: alkaloid analysis. II. Rev. Biol. Trop. 13(2): 207-212.

349.Sáenz, J.A., Nassar, M. \& Sáenz, G.V. 1981. Phytochemical screening of Costa Rican plants: Alkaloid analysis. VI. Rev. Biol. Trop. 29(2): 283293.

350.Salas Murillo, H. 1969. Derivados antracénicos en Rumex crispus L. y en Rumex obtusifolius L. Tesis de Licenciatura en Farmacia, Universidad de Costa Rica.

351.Salas, I. 1985. Mecanismo de acción cardiovascular de los extractos frescos liofilizados de Cecropia obtusifolia Bertol. Tesis de Maestría en Ciencias Biomédicas, Universidad de Costa Rica.

352.Salas, I., Brenes, J.R. \& Morales, M. 1987. Antihypertensive effect of Cecropia obtusifolia (Moraceae) leaf extract on rats. Rev. Biol. Trop. 35(1): 127-130.

353.Salas, J. 1994. Actividad cicatrizante del látex de Jatropha curcas (Angiospermae, Euphorbiaceae). Rev. Biol. Trop. 42(1-2): 323-326.

354.Salas, S. 1951. Estudio del látex de Brosimum utile (mastate). Tesis de Licenciatura en Farmacia, Universidad de Costa Rica.

355.Sánchez Borbón, H. 1953. Investigación de alcaloides y glucósidos en la cabalonga. Tesis de Licenciatura en Farmacia, Universidad de Costa Rica.

356. Sánchez Guzmán, O. 1969. Contribución al estudio fitoquímico de especies costarricenses del género Datura. Tesis de Licenciatura en Farmacia, Universidad de Costa Rica.

357.Sánchez Rojas, B. 1944. Algunos datos experimentales sobre el uso de la cabalonga como medicamento para combatir la malaria o el paludismo. Tesis de Licenciatura en Farmacia,
Universidad de Costa Rica.

358.Sánchez Umaña, M. 1963. Algunas experiencias sobre la acción farmacológica de la planta Gliricidia sepium Steud., conocida en Costa Rica como madero negro. Tesis de Licenciatura en Farmacia, Universidad de Costa Rica.

359. Sancho B., A. 1957. Comparación del valor alimenticio de la zanahoria y la arracacha. Tesis de Licenciatura en Farmacia, Universidad de Costa Rica.

360.Sancho Bolaños, D. 1958. Rauwolfia canescens var. glabra. Investigación del alcaloide reserpina en su raíz. Tesis de Licenciatura en Farmacia, Universidad de Costa Rica.

361.Sarkis, S.A. \& Campos M., V.M. 1952. Medicamentos populares de la Meseta Central y la zona atlántica, comprendiendo además medicina y costumbres de los indígenas y de los habitantes de color. Tesis de Licenciatura en Farmacia, Universidad de Costa Rica.

362.Sarkis, S.A. \& Campos, M.V. 1985. Curanderismo tradicional del costarricense. San José, Edit. Costa Rica.

363.Scott Davis, C.N. 1958. Contribución al estudio de los principios activos de la Scoparia dulcis (escobilla blanca o mastuerzo). Tesis de Licenciatura en Farmacia, Universidad de Costa Rica.

364.Segleau Earle, J. 2001. Plantas medicinales en el trópico húmedo. San José, Edit. Guayacán.

365. Sibaja, M. \& Durán, M. 1986. Modificación química de la celulosa de piña. Obtención del acetato de celulosa. Ing. Cie. Quím. 10(3-4): 5153.

366.Sibaja, M., Durán, M., Bolaños, R. \& Mata, J. 1982. Estudio morfológico preliminar de la fibra cruda y deslignizada de piña (Ananas comosus). Ing. Cie. Quím. 6(2):183-184.

367.Siebert, S.F. 2000. Primary forests and medicinal plants. Conserv. Biol. 14(3): 596.

368.Solano Hernández, J.M. 1963. Estudio químico bromatológico de las hojas comestibles de la acedera (Rumex acetosa L.). Tesis de Licenciatura en Farmacia, Universidad de Costa Rica.

369. Soley C., R. 1941. Investigación de rotenona en algunas especies de Lonchocarpus y Cracca de Costa Rica. Tesis de Licenciatura en Farmacia, Universidad de Costa Rica.

370. Soto Córdoba, S.M. 1994. Evaluación fitoquímica del follaje de Gliricidia sepium (familia Leguminosae). Tesis de Licenciatura en Química, Universidad de Costa Rica. 
371.Soto Fallas, R. 1989. Estudio fitoquímico de las hojas de Phoebe cinnamomifolia (H.B.K.) Nees. Tesis de Licenciatura en Química, Universidad de Costa Rica.

372.Soto Fallas, R.M. 1990. Estudio fitoquímico de las hojas de Phoebe cinnamomifolia (H.B.K.) Nees. Cie. Tecnol. 14(1-2): 223.

373.Suárez U., A. 1995. Efectos generales y cardiovasculares producidos por extractos etanólico, acuoso y sus fracciones de Pimenta dioica (L.) Merrill en ratas albinas normotensas e hipertensas. Tesis de Maestría en Ciencias Biomédicas, Universidad de Costa Rica.

374.Suárez U. A., Ulate Montero, G. \& Cicció Alberti, J.F. 1996-1997. Efectos de la administración aguda y subaguda de extractos de Pimenta dioica (Myrtaceae) en ratas albinas normotensas e hipertensas. Rev. Biol. Trop. 44(3)/45 (1): 39-45.

375.Suárez U., A., Ulate Montero, G. \& Cicció Alberti, J.F. 2000. Hypotensive action of an aqueous extract of Pimenta dioica (Myrtaceae) in rats. Rev. Biol. Trop. 48(1): 53-58.

376.Surgeon B., J.L. 1945. La súrtuba amarga. Tesis de Licenciatura en Farmacia, Universidad de Costa Rica.

377.Tapia Rodríguez, J.A. 1967. Detección rápida de alcaloides, utilizando cromatografía de capa delgada. Tesis de Licenciatura en Farmacia, Universidad de Costa Rica.

378.Torres Bonilla, A.A. 1994. Estudio básico para la producción de acetona-butanol, vía fermentativa, utilizando como sustrato banano verde. Tesis de Licenciatura en Ingeniería Química, Universidad de Costa Rica.

379.Torres Chinchilla, L.I. 1997. Determinación de la actividad anti-inflamatoria de extractos de Urera caracasana y comparación de dos equipos de medición del modelo de edema de pata. Tesis de Licenciatura en Farmacia, Universidad de Costa Rica.

380.Torres Peñaranda, A.V. 1984. Estudio químico preliminar sobre la calidad de la lima, Citrus aurantifolia cultivar mesina. Tesis de Licenciatura en Tecnología de Alimentos, Universidad de Costa Rica.

381.Trimiño Vásquez, H. 1975. Obtención de proteína a partir de banano. Tesis de Licenciatura en Química, Universidad de Costa Rica.

382.Umaña Rojas, E. 1990. Estudio fitoquímico de la parte aérea de Verbena litoralis. Tesis de Licenciatura en Química, Universidad de Costa Rica.
383.Umaña Solano, C. 1951. Gunzo (Scheelea rostrata Burr.). Estudio de esta palma y de los aceites contenidos en sus frutos. Tesis de Licenciatura en Farmacia, Universidad de Costa Rica.

384.Umaña Umaña, C. 1961. Ensayo fitoquímico de la especie Stemmadenia glabra Benth. (guijarro). Tesis de Licenciatura en Farmacia, Universidad de Costa Rica.

385.Urbina Campos, A. 1985. Alcaloides de las ramitas de la especie Tabernaemontana arborea. Tesis de Licenciatura en Química, Universidad de Costa Rica.

386.Valverde Cerdas, J. 1994. Búsqueda de componentes con actividad antibacteriana en dos especies de la familia Rubiaceae. Tesis de Licenciatura en Química, Universidad de Costa Rica.

387.Vallejo Esquivel, E.A. 1996. Evaluación de la actividad diurética y antimicrobiana de dos plantas usadas como diuréticos en Costa Rica. Tesis de Licenciatura en Farmacia, Universidad de Costa Rica.

388.Van der Laat, J.E. 1951. Estudio comparativo del contenido de ácido cítrico y vitamina $\mathrm{C}$ en el jugo de algunas variedades de Citrus de uso popular. Tesis de Licenciatura en Farmacia, Universidad de Costa Rica.

389.Van der Laat, J.E. 1954. Estudio comparativo del contenido de ácido cítrico y vitamina $\mathrm{C}$ en el jugo de algunas variedades de Citrus de uso popular. Rev. Biol. Trop. 2(1): 45-58.

390. Varela Morales, M. \& Vindas Durán, R. 1977. Contenido alcaloidal de Lobelia laxiflora. Tesis de Licenciatura en Farmacia, Universidad de Costa Rica.

391.Vargas B., A. 1952. Investigación de la ureasa en los frijoles más corrientes de Costa Rica. Tesis de Licenciatura en Farmacia, Universidad de Costa Rica.

392.Vargas Bejarano, S. 1942. Investigación de carotenos en maíz amarillo y el pejibaye. Tesis de Licenciatura en Farmacia, Universidad de Costa Rica.

393.Vargas Carranza, J.L. 1989. Talamanca; la ocupación aborigen del medio ambiente: aportes para un desarrollo sostenible y duradero. Vínculos 15(1-2): 69-83.

394.Vargas Chinchilla, S. 1990. Plantas medicinales: la naturaleza como guardián de su salud. $2^{\mathrm{a}}$. ed. San José, Argüello Rosales.

395.Vargas Howell, R. \& Ulate Montero, G. 1958. 
Actividad diurética de la Cecropia obtusifolia (Moraceae) en ratas albinas. Rev. Biol. Trop. 44(1): 93-96.

396.Vila, R., Iglesias, J., Cañigueral, S. \& Cicciò, J.F. 2000. Essential oil of Tagetes filifolia Lag. from Costa Rica. Ing. Cie. Quím. 19: 13.

397.Villalobos del Valle, C. 1951. El sagú en Costa Rica (Maranta arundinacea L.). Tesis de Licenciatura en Farmacia, Universidad de Costa Rica.

398.Villalobos Miranda, R. 1962. Contribución al estudio de la planta Eugenia jambos L., conocida en Costa Rica vulgarmente con el nombre de manzana rosa. Tesis de Licenciatura en Farmacia, Universidad de Costa Rica.

399.Villalobos Soto, R. 1996. Caracterización de la distribución de una planta medicinal (Quassia amara) como base para su manejo técnico. In: Bertsch, F., Badilla, W. \& García, J. (eds.). Memoria: ¿Puede la agricultura sostenible ser competitiva? Agronomía y Recursos Naturales. 10. Congreso Nacional Agronómico y de Recursos Naturales; 3. Congreso Nacional de Fitopatología; 2. Congreso Nacional de Suelos. 8-12 jul., San José. Asociación Costarricense de la Ciencia del Suelo; Colegio de Ingenieros Agrónomos; Asociación Costarricense de Fitopatólogos. EUNED/EUNA. p. 17-22.

400.Villalobos V., J. et al. 1989. Trabajar como mujeres: nuestra historia. Producción de Mujeres Unidas de Sarapiquí (MUSA). ILPEC, ACECAN. (Videocasete)

401.Villalobos, R. 2000. Cuculmeca y zarzaparrilla: plantas medicinales típicas con problemas de uso típicos de América tropical. Rev. For. Centroamer. (CATIE) 31: 39-42.

402.Villalobos, R., Chang, Y., Marmillod, D., Bedoya, R. \& Leigue, L. 1997. Desarrollo de criterios silviculturales para el manejo de Quassia amara, un producto no maderable del bosque tropical. Simposio Internacional sobre Posibilidades de Manejo Forestal Sostenible en América Tropical. Santa Cruz de la Sierra, Bolivia, 15-20 jul. Proyecto de Manejo Forestal Sostenible; CIFOR. p. 64-70.

403.Víquez Carazo, A. 1958. Investigación de la acción vermífuga de tres especies de Ficus que crecen en Costa Rica. Tesis de Licenciatura en Farmacia, Universidad de Costa Rica.

404. Vives Gifre, L. 1942. El limón agrio en Costa Rica. Tesis de Licenciatura en Farmacia, Universidad de Costa Rica.
405.Wainberg Konskier, G. 1983. Evaluación de la calidad de Mentha sp. Tesis de Licenciatura en Tecnología de Alimentos, Universidad de Costa Rica.

406.Wardle, R. 2001. A community based case study of medicinal plant knowledge in southern Costa Rica. Thesis of Master in Environmental Studies. York University, Ontario, Canada. 93 p.

407. Watson Samudio, H. 1991. Evaluación fitoquímica de las hojas de Senna alata y Cassia fruticosa. Tesis de Maestría en Química, Universidad de Costa Rica.

408.Zamora A., M.R. 1954. Propiedades lactagogas del ixbut. Tesis de Licenciatura en Farmacia, Universidad de Costa Rica.

409.Zavala Ortega, W.E. 1990. Estudio de las características de curtientes del nance (Byrsonima crassifolia) de Costa Rica. Cie. Tecnol. 14(1-2): 219.

410.Zavaleta M., R. 1968. Estudio analítico de la cera obtenida de la Myrica cerifera (arrayán). Tesis de Licenciatura en Farmacia, Universidad de Costa Rica.

411.Zeledón Araya, A. 1958. Determinación de un posible principio analgésico en las hojas del mango (Mangifera indica L.). Tesis de Licenciatura en Farmacia, Universidad de Costa Rica.

412.Zeledón Castro, J.B. 1946. Algunos datos sobre Artocarpus communis Forst. (Artocarpus incisa (Thunb.) L.f.), fruta de pan, y análisis de su valor alimenticio. Tesis de Licenciatura en Farmacia, Universidad de Costa Rica.

413.Zorn, García, Castro, Murillo, Mora \& Merfort. 2001. 3-desoxyanthocyanidins from Arrabidaea chica (Bignoniaceae). Phytochemistry 54: 831.

414.Zumbado, M. \& Murillo, M. 1984. Composition and nutritive value of pejibaye (Bactris gasipaes) in animal feeds. Rev. Biol. Trop. 32(1): 51-56.

415.Zúñiga Loría, E. 2001. Efecto de las cápsulas de nopal (Opuntia indica, Opuntia streptacantha) y té de hierbas sobre el crecimiento y diferentes parámetros bioquímicos en ratas SpragueDawley. Práctica dirigida de Licenciatura en Microbiología y Química Clínica, Universidad de Costa Rica.

416.Zúñiga O., F. 1944. Vitamina C en el jocote. Tesis de Licenciatura en Farmacia, Universidad de Costa Rica. 


\section{ÍNDICE DE FAMILIAS, GÉNEROS Y ESPECIES DE LAS REFERENCIAS}

Este índice incluye sobre todo estudios fitoquímicos, farmacológicos y bromatológicos; es decir, aquéllos que proporcionan información científica sobre la composición química y las propiedades de las plantas. En todos los casos, se trata de plantas vasculares, divididas en Pteridófitos (Pteridophyta) y Espermatófitos (Spermatophyta); éstos, a su vez, se agrupan en Gimnospermas y Angiospermas; éstas se dividen en Dicotiledóneas y Monocotiledóneas. Las familias de cada grupo aparecen en orden alfabético. En lo posible, se ha actualizado la nomenclatura y, cuando necesario, se han agregado sinónimos que se hallan en la literatura. Después de los nombres científicos se anotan los números de referencias, además de los nombres comunes (cuando éstos se conocen). Debe quedar claro que no se trata solamente de plantas

\section{PTERIDOPHYTA}

Cyatheaceae

Cyathea arborea (L.) Sm. 300 (rabo de mico)

SPERMATOPHYTA

\section{GyMnOSPERMAE}

Cupressaceae

Cupressus lusitanica Mill. 349 (ciprés)

\section{Zamiaceae}

Zamia skinneri Warsz. ex A.Dietr. 140 (zamia)

Zamia sp. 140 [la obra citada indica "Z. loddigesii

Miq.", pero este nombre no corresponde a ninguna especie (sp.) de Costa Rica]

\section{ANGIOSPERMAE}

\section{DICOTILEDÓNEAS}

Acanthaceae

Blechum brownei Juss. [= B. pyramidatum (Lam.) Urb.] 140

Dicliptera unguiculata Nees 345 (sornia, olotillo) Justicia aurea Schltdl. [= Jacobinia umbrosa (Benth.) S.F.Blake] 348

Justicia tinctoria (Oerst.) D.N.Gibson [Jacobinia tinctoria (Oerst.) Hems1.] 151, 348 (azul de mata)

Actinidiaceae ( Saurauiaceae)

Saurauia montana Seem. (= S. costaricensis Donn. medicinales.

Muchos investigadores no prepararon especímenes testigo de las plantas estudiadas, para ser depositados en un herbario reconocido. Por esta razón, en algunos casos el nombre científico dado por el autor de un estudio es dudoso; en otras palabras, no podemos estar seguros de que la planta mencionada en el texto es la que realmente se investigó. Este es uno de los aspectos que deben cambiar para siempre en el estudio de las plantas medicinales. Por último, no hemos incluido aquí un índice de nombres comunes de plantas medicinales, porque un estudio serio de estas plantas sólo es posible con una determinación segura de las especies, con sus nombres científicos correctos y ejemplares testigo en herbarios.

\section{Sm.) 345,349 (moco)}

\section{Aizoaceae}

Sesuvium portulacastrum L. 343

Amaranthaceae

Alternanthera amoena Back. \& Sloot. 345

Amaranthus hybridus L. 90 (amaranto, bledo blanco) Iresine diffusa Humb. \& Bonpl. ex Willd. (= I. celosia L.) 345 (camarón)

Anacardiaceae

Anacardium excelsum (Bertero \& Balb.) Skeels [= A. rhinocarpus (Bertero \& Balb.) DC.] 192, 345 (espavel)

Astronium graveolens Jacq. (= A. fraxinifolium Schott ex Spreng.) 349 (ronrón)

Mangifera indica L. 341, 411 (mango)

Mauria heterophylla Kunth (= M. biringo Tul.) 343 (lantisco, cirrí)

Spondias mombin L. 345 (jobo)

Spondias purpurea L. 91, 334, 349, 416 (jocote)

Tapirira mexicana March. (= T. brenesii Standl.) 283 (cirrí, ciruelo de monte)

\section{Annonaceae}

Annona cherimola Mill. 349 (anona, chirimoya)

Annona muricata L. 111, 341 (guanábana)

Annona reticulata L. 245, 349 (anona)

Desmopsis oerstedii Saff. 245 (guineo)

Guatteria diospyroides Baill. 194 (limoncillo)

Sapranthus palanga R.E.Fries 345 (palanco, guineo) 
Xylopia sericophylla Standl. 345 (manga larga)

Apiaceae (= Umbelliferae)

Arracacia xanthorrhiza Bancr. var xanthorrhiza 308, 359 (arracacha, arracache) [Algunos autores han usado el nombre $A$. atropurpurea (Lehm.) Benth. \& Hook.f. ex Hemsl.]

Coriandrum sativum L. 219 (culantro, c. de Castilla)

Daucus carota L. 359 (zanahoria)

Eryngium carlinae F.Delaroche 341

Eryngium foetidum L. 219 (culantro de coyote)

Myrrhidendron donnell-smithii J.M.Coult. \& Rose 292, 348, 349 (arracachillo)

Pimpinella anisum L. 337 (anís)

Apocynaceae

Allamanda cathartica L. 141, 295, 341 (bejuco de San José)

Allamanda schottii Pohl (= A. neriifolia Hook.) 110

Catharanthus roseus (L.) G.Don (Vinca rosea L.) 377 (pervinca)

Lacmellea panamensis (Woodson) Markgr. 140 (lagarto)

Mandevilla subsagittata (Ruiz \& Pav.) Woodson 345

Nerium oleander L. 295 (adelfa, "narciso")

Peschiera arborea (Rose) Markgr. (Tabernaemontana arborea Rose ex Donn.Sm.) 52, 83, 103, 106, 110, 385

Rauvolfia sarapiquensis Woodson 113 (también se ha escrito como Rauwolfia)

Rauvolfia tetraphylla L. (= R. canescens L., R. hirsuta Jacq.) 343, 360 (cuataco, coataco)

Stemmadenia alfari (Donn.Sm.) Woodson 221 (guijarro)

Stemmadenia donnell-smithii (Rose) Woodson 140 (huevos de caballo)

Stemmadenia litoralis (Kunth) L.Allorge $[=S$. galeottiana (A.Rich.) Miers] 140 (huevos de caballo)

Stemmadenia obovata (Hook. \& Arn.) K.Schum. (= S. glabra Benth.) 79, 84, 140, 341, 348, 377, 384 (huevos de caballo, guijarro)

Stemmadenia spp. 81, 85

Tabernaemontana longipes Donn.Sm. 110

Thevetia ovata (Cav.) A.DC. [=Thevetia plumeriaefolia Benth., Cascabela ovata (Cav.) Lippold] 244, 341, 342, 345 (chirca venenosa)

Thevetia peruviana (Pers.) K.Schum. (= T. neriifolia Juss.) 250, 343 (chirca)

Aquifoliaceae

Ilex lamprophylla Standl. 345
Araliaceae

Dendropanax arboreus (L.) Decne. \& Planch. 245 (cacho de venado)

Oreopanax capitatus (Jacq.) Decne. \& Planch. 349 (cacho de venado)

Oreopanax xalapensis (Kunth) Decne. \& Planch. 341 (cacho de venado)

Sciadodendron excelsum Griseb. 245 (chile)

Asclepiadaceae

Asclepias curassavica L. 341 (viborana)

Gonolobus edulis Hemsl. [Vincetoxicum edule (Hemsl.)

Stand1.] 23, 341 (cuayote)

Marsdenia brenesii Standl. 343

Asteraceae (= Compositae) 86

Acmella alba (L'Her.) R.K.Jansen [Spilanthes alba L'Her., S. ocymifolia (Lam.) A.H.Moore] 86

Ageratina anisochroma (Klatt) R.M. King \& H.Rob.

(Eupatorium anisochromum Klatt) 343

Ageratina cartagoensis R.M. King \& H.Rob. 224

Ageratina ligustrina (DC.) R.M.King \& H.Rob.

(Eupatorium ligustrinum DC.) 86, 325, 326, 327

Archibaccharis schiedeana (Benth.) J.D.Jackson 86

Arctium lappa L. 337 (bardana)

Artemisia absinthium L. 337 (ajenjo)

Baccharis braunii (Polak.) Standl. 343

Baccharis pedunculata (Mill.) Cabrera 86

Baccharis trinervis Pers. [Pseudobaccharis trinervis

(Pers.) V.M.Badillo] 86

Baltimora recta L. 348

Bidens pilosa L. 86

Bidens squarrosa Kunth 86

Calea urticifolia (Mill.) DC. 345 (jaral, jalacate)

Calyptocarpus vialis Less. 345 (espinillo)

Chaptalia nutans (L.) Pol. 26, 112 (árnica)

Chromolaena glaberrima (DC.) R.M.King \& H.Rob.

(Eupatorium glaberrimum DC.) 86

Cichorium endivia L. 63 (escarola)

Clibadium aff. schulzii S.F.Blake 86

Critonia hebebotrya DC. [Eupatorium hebebotryum (DC.) Hemsl.] 348

Decachaeta thieleana (Klatt) R.M.King \& H.Rob. 86 Delilia biflora (L.) Kuntze (=D. berteroi Spreng.) 86

Echinacea purpurea (L.) Moench 99, 168, 301 (equinácea)

Emilia sonchifolia (L.) DC. 341

Galinsoga quadriradiata Ruiz \& Pav. [= G. urticaefolia

(Kunth) Benth.] 86

Gnaphalium attenuatum DC. 86

Helianthus annuus L. 109 (girasol)

Hypochoeris radicata L. 86 
Jaegeria hirta (Lag.) Less. 86

Koanophyllum pittieri (Klatt) R.M.King \& H.Rob. (Eupatorium pittieri Klatt) 348

Lactuca sativa L. 313 (lechuga)

Lasianthaea fruticosa (L.) K.M.Becker 86

Lycoseris latifolia (D.Don) Benth. 64, 208

Matricaria chamomilla $\mathrm{L}$. [= Chamomilla recutita $(\mathrm{L}$. Rauschert] 40, 44, 46, 126, 154, 164 (manzanilla)

Melampodium divaricatum (Rich.) DC. [= M.flaccidum (Benth.) Oerst.] 86

Melampodium perfoliatum (Cav.) Kunth 86

Melanthera nivea (L.) Small 86

Mikania guaco Humb. \& Bonpl. 3, 168 (guaco)

Montanoa atriplicifolia (Pers.) Sch.Bip. (= M. dumicola Klatt) 104

Montanoa hibiscifolia Benth. 54

Neurolaena lobata (L.) R.Br. 86, 233 (gavilana)

Onoseris onoseroides (Kunth) B.L.Rob. (= O. silvatica Greenm.) 86

Oyedaea verbesinoides DC. 86

Pentacalia andicola (Turcz.) Cuatrec. (Senecio andicola Turcz.) 343

Podachaenium eminens (Lag.) Sch.Bip. 65

Sclerocarpus divaricatus (Benth.) Benth. \& Hook.f. 86

Senecio costaricensis R.M. King 328, 329, 332

Senecio multivenius Benth. 348

Senecio oerstedianus Benth. 328, 329, 333, 348

Smalanthus maculatus (Cav.) H.Rob. (Polymnia maculata Cav.) 348

Sonchus oleraceus L. 341 (diente de león)

Synedrella nodiflora (L.) Gaertn. 86 (espinillo)

Tagetes erecta L. 341

Tagetes filifolia Lag. 225, 396 (anisillo)

Tagetes foetidissima DC. 141 (flor de muerto)

Tagetes microglossa Benth. (= T. jaliscensis Greenm.) 59, 235 (rodillo)

Tagetes spp. 235

Tanacetum parthenium (L.) Sch.Bip. [Chrysanthemum parthenium (L.) Bernh.] 337 (altamisa, artemisa)

Tithonia diversifolia (Hemsl.) A.Gray 86, 338

Tithonia rotundifolia (Mill.) S.F.Blake 86

Tridax procumbens L. 86

Verbesina tonduzii Greenm. 142, 196 (capitana)

Verbesina turbacensis Kunth 86

Vernonia canescens Kunth 21 (tuete)

Vernonia patens Kunth 86, 140, 168, 301 (tuete)

Vernonia stellaris La Llave \& Lex. 341

Avicenniaceae ( Verbenaceae)

Avicennia germinans (L.) L. (= A. nitida Jacq.) 345 (palo de sal)
Basellaceae

Anredera ramosa (Moq.) Eliasson [Boussingaultia ramosa (Moq.) Hemsl.] 343

Betulaceae

Alnus acuminata Kunth 348 (jaúl)

Bignoniaceae

Anemopaegma chrysoleucum (Kunth) Sandw. (=A. puncticulatum Pittier \& Standl.) 343

Arrabidaea chica (Humb. \& Bonpl.) Verl. 413

Crescentia cujete L. 273, 343 (jícaro, raspaguacal)

Godmania aesculifolia (Kunth) Standl. 349 (corteza de chivo)

Jacaranda caucana Pittier (= J. filicifolia D.Don) 343 (jacaranda)

Mansoa hymenaea (DC.) A.H.Gentry [= Pseudocalymma macrocarpum (Donn.Sm.) Sandw.] 345 (hosmeca, jumeca)

Spathodea campanulata Beauv. 343, 348 (llama del bosque)

Tecoma stans (L.) Juss. ex Humb. 343 (vainillo)

Bixaceae

Bixa orellana L. 14, 15, 24, 141, 341 (achiote, achote)

Bombacaceae

Ceiba aesculifolia (Kunth) Britten \& Baker f. 245 (pochote pelota)

Ochroma pyramidale (Cav. ex Lam.) Urb. (= O. lagopus Sw.) 343 (balsa)

Boraginaceae

Borago officinalis L. 337 (borraja)

Cordia alliodora (Ruiz \& Pav.) Cham. 345 (laurel)

Cordia collococca L. $(=C$. collococca L. var. micrantha, C. micrantha Sw., C. glabra L.) 186, 341 (muñeco)

Cordia eriostigma Pittier (mal identificada como $C$. glabra L.) 245 (muñeco)

Cordia lasiocalyx Pittier 349

Heliotropium indicum L. 341

Tournefortia bicolor Sw. 345

Tournefortia glabra L. 341

Brassicaceae (= Cruciferae)

Brassica oleracea L. var. capitata 36, 63, 313 (repollo, r. morado)

Brassica sp. 313 (mostaza)

Lepidium virginicum L. (= L. costaricense Thell.) 345 (mastuerzo)

Nasturtium officinale R.Br. 63, 313, 337 (berro) 
Raphanus sativus L. 63 (rábano)

Brunelliaceae

Brunellia costaricensis Standl. 349 (cedrillo)

Buddlejaceae ( Loganiaceae)

Buddleja americana L. 168, 301 (salvia virgen)

Burseraceae

Boswellia sp. 140 (incienso)

Bursera simaruba (L.) Sarg. 140, 341 (jiñocuabe, indio desnudo)

Bursera tomentosa (Jacq.) Triana \& Planch. 245

Commiphora sp. 140 (mirra)

Protium sp. 140 (copal, canfín)

Cactaceae

Opuntia ficus-indica (L.) Mill. 415 (tuna, nopal) (en la obra citada, nombre mal escrito como "O. indica")

Opuntia streptacantha Lem. 415

Campanulaceae ( Lobeliaceae)

Hippobroma longiflora (L.) G.Don [Isotoma longiflora (L.) C.Presl] 345 (jazmín de estrella, jazmincillo)

Lobelia laxiflora Kunth 227, 343, 390 (tabaco indio, ceragallo)

Capparidaceae

Capparis incana Kunth 245, 349

Capparis indica (L.) Druce 348

Capparis odoratissima Jacq. 245

Caprifoliaceae

Sambucus canadensis L. (= S. oreopola Donn.Sm.) 348

Caricaceae

Carica papaya L. (= C. peltata Hook. \& Arn.) 153, 206, 348 (papaya)

Cecropiaceae ( Moraceae)

Cecropia obtusifolia Bertol. (= C. mexicana Hemsl.) 348, 351, 352, 395 (guarumo)

Cecropia polyphlebia Donn.Sm. 349 (guarumo)

Coussapoa villosa Poepp. \& Endl. (= C. panamensis Pittier) 245

Celastraceae

Crossopetalum tonduzii (Loes.) Lundell (= Gyminda costaricensis Standl.) 73, 105, 218

Zinowiewia costaricensis Lundell 72
Chenopodiaceae

Beta vulgaris L. 213 (remolacha)

Chenopodium ambrosioides L. 290, 309 (apazote, té de México)

Spinacia oleracea L. 63, 165, 313 (espinaca)

Chloranthaceae

Hedyosmum spp. 234

Chrysobalanaceae ( Rosaceae)

Chrysobalanus icaco L. 49 (icaco)

Licania arborea Seem. 152 (alcornoque, roble blanco)

Clethraceae

Clethra lanata M.Martens \& Galeotti 343 (nance macho)

Clusiaceae (= Guttiferae)

Clusia alata Planch. \& Triana 348

Clusia amazonica Planch. \& Triana $(=$ C. oedematopoidea Maguire) 157

Clusia coclensis Standl. 130, 132, 133, 135, 157

(copey, azahar de monte)

Clusia flava Jacq. 34, 157 (copey)

Clusia gracilis Standl. 157

Clusia liesneri Maguire 157

Clusia minor L. (= C. odorata Seem.) 157, 345 (azahar de monte)

Clusia palmana Standl. 157

Clusia quadrangula Bartlett 157

Clusia rosea Jacq. 157 (algunos autores han usado el nombre L. major L.)

Clusia rotundata Standl. 157

Clusia salvinii Donn.Sm. 157

Clusia stenophylla Stand1. 34, 157

Clusia torresii Standl. 157

Clusia uvitana Pittier (= C. erectistigma Maguire) 157

Clusia valerii Standl. 157

Clusia sp. 157 (la obra citada indica "C. guanacastensis Standl.”, un nombre que no aparece publicado)

Garcinia intermedia (Pittier) Hammel $[=$ Rheedia edulis (Seem.) Triana \& Planch.] 245 (jorco)

Symphonia globulifera L.f. 345 (cerillo, botoncillo)

Vismia guianensis (Aubl.) Pers. 348

Cochlospermaceae

Cochlospermum vitifolium (Willd.) Spreng. 191 (poro-poro)

Combretaceae

Conocarpus erecta L. 348 (mangle torcido, m. 
mariquita)

Terminalia catappa L. 2, 150, 345 (almendro de playa)

Convolvulaceae

Ipomoea alba $\mathrm{L}$. [= Calonyction aculeatum (L.) House] 343 (flor de luna)

Ipomoea batatas (L.) Lam. 313, 343 (camote)

Ipomoea jalapa (L.) Pursh [Exogonium jalapa (L.)

Baill., Convolvulus jalapa L.] 335

Ipomoea neei (Spreng.) O'Donnell 245

Ipomoea parasitica (Kunth) G.Don 230 (churristate, rompeorejas)

Ipomoea pes-caprae (L.) R.Br. 348 (pie de cabra)

Ipomoea purpurea (L.) Roth 122 (churristate, campanilla)

Coriariaceae

Coriaria thymifolia Humb. \& Bonpl. 343

Cucurbitaceae

Cucumis sativus L. 313 (pepino)

Cucurbita pepo L. 313, 341 (ayote)

Cucurbita sp. 274 (pipián)

Fevillea cordifolia L. 66, 355, 357 (cabalonga)

Luffa acutangula (L.) Roxb. 76 (paste, esponja vegetal)

Luffa aegyptiaca Mill. (= Luffa cylindrica M.Roem.) 345 (paste, estopa)

Momordica charantia L. 282 (sorosí, sorisí)

Sechium edule (Jacq.) Sw. 63, 341 (chayote)

Sechium tacaco (Pittier) C.Jeffrey [Frantzia tacaco

(Pittier) Wunderlin, Polakowskia tacaco Pittier] 9, 348 (tacaco)

Cunoniaceae

Weinmannia pinnata L. 343 (lorito)

Dichapetalaceae

Dichapetalum donnell-smithii Engl. 349

Ebenaceae

Diospyros nicaraguensis Standl. 349

Elaeagnaceae

Elaeagnus parvifolia Wall. ex Royle $(=$ E. umbellata

Thunb.) 348

Elaeocarpaceae

Sloanea zuliaensis Pittier (= S. macropoda Stand1.) 349

Ericaceae

Gaultheria erecta Vent. [= G. costaricensis (Donn.
Sm.) Small] 348

Gaultheria gracilis Small 8

Pernettya prostrata (Cav.) DC. (= P. coriacea Klotzsch) 217, 344, 348 (arrayán)

Vaccinium consanguineum Klotzsch 348 (arrayán)

Vaccinium poasanum Donn.Sm. 349 (arrayán)

Erythroxylaceae

Erythroxylum havanense Jacq. 245, 349

Erythroxylum lucidum Kunth 245, 349

Euphorbiaceae

Acalypha sp. 140 (cafecillo, ortiga)

Acalypha alopecuroidea Jacq. 140

Acalypha diversifolia Jacq. 349

Acalypha macrostachya Jacq. 349

Alchornea latifolia Sw. 349

Chamaesyce hyssopifolia (L.) Small 140

Cnidoscolus aconitifolius (Mill.) I.M.Johnst. (Jatropha aconitifolia Mill.) 116, 341 (chicasquil)

Cnidoscolus tubulosus (Müll.Arg.) I.M. Johnst. (Jatropha tubulosa Müll.Arg.) 345 (yerba santa)

Croton decalobus (Benth. \& Hook.) Müll.Arg. 349

Croton draco Cham. \& Schltdl. (= C. gossypiifolius Vahl) 195, 341 (targuá)

Croton hirtus L'Her. 125

Croton jimenezii Standl. \& Valerio 239

Croton niveus Jacq. 245, 341 (copalchí)

Croton xalapensis Kunth 237, 349 (targuá blanco)

Euphorbia aucheri Boiss. 238

Euphorbia cotinifolia L. 140, 141, 341 (barrabás, urrú)

Euphorbia lancifolia Schltdl. 408 (ixbut)

Gymnanthes lucida Sw. 245

Hieronyma poasana Standl. 349

Hura crepitans L. 140 (javillo)

Jatropha costaricensis G.L.Webster \& Poveda 110

Jatropha curcas L. 353 (tempate, coquito)

Jatropha gossypiifolia L. 141, 341 (frailecillo)

Mabea montana Müll.Arg. 345

Manihot esculenta Crantz [= M. utilissima Pohl, $M$. dulcis (J.F.Gmelin) Pax, M. palmata Müll.Arg.] 2, 49, 287 (yuca, yuca dulce, mandioca, mañoco)

Pedilanthus tithymaloides (L.) Poit. 140 (titímalo)

Pera aff. arborea Mutis 349

Phyllanthus anisolobus Müll.Arg. (= P. pittieri Pax) 245

Ricinus communis L. 341 (higuerilla, ricino)

Sapium macrocarpum Müll.Arg. 245, 349 (yos)

Sapium pachystachys K.Schum. \& Pittier 245 (yos)

Tetrorchidium euryphyllum Standl. 349 
Fabaceae (= Leguminosae, Caesalpiniaceae, Mimosaceae, Papilionaceae)

Subfamilia Caesalpinioideae

Bauhinia sp. 341

Bauhinia guianensis Aubl. (= B. manca Standl.) 118, 140 (escalera de mono)

Bauhinia pauletia Pers. 345 (pie de venado)

Caesalpinia exostemma DC. [= C. conzattii (Rose) Standl.] 349

Cassia fistula L. 140 (caña fístula)

Cassia grandis L.f. 181, 324 (carao)

Haematoxylon brasiletto H.Karst. 245 (palo de tinta)

Hymenaea courbaril L. 306, 320, 341 (guapinol)

Peltogyne purpurea Pittier 345 (nazareno)

Prioria copaifera Griseb. 5 (cativo, camíbar)

Senna alata (L.) Roxb. (Cassia alata L.) 149, 311, 407 (saragundí)

Senna bacillaris (L.f.) H.S.Irwin \& Barneby (Cassia bacillaris L.f.) 348

Senna fruticosa (Mill.) H.S.Irwin \& Barneby (Cassia fruticosa Mill.) 407

Senna nicaraguensis (Benth.) H.S.Irwin \& Barneby (Cassia nicaraguensis Benth.) 311

Senna reticulata (Willd.) H.S.Irwin \& Barneby (Cassia reticulata Willd.) 216, 311, 341 (saragundí)

Senna spectabilis (DC.) H.S.Irwin \& Barneby (Cassia spectabilis DC.) 140, 341, 349 (vainillo)

Senna spp. (= Cassia spp.) 311

Tamarindus indica L. 220, 348 (tamarindo)

Subfamilia Mimosoideae

Acacia angustissima (Mill.) Kuntze (= A. pittieriana Standl.) 341 (carboncillo)

Acacia centralis (Britton \& Rose) Lundell [antes se usó el nombre Lysiloma desmostachyum (Benth.) Benth.] 343 (ardillo)

Acacia cornigera (L.) Willd. (= A. spadicigera Schltdl. \& Cham.) 343 (cornezuelo)

Acacia tenuifolia (L.) Willd. 345

Calliandra calothyrsus Meisn. (= C. confusa Sprague \& Riley) 348

Cojoba costaricensis Britton \& Rose [Pithecellobium costaricense (Britton \& Rose) Standl.] 245, 349 (lorito)

Enterolobium cyclocarpum (Jacq.) Griseb. 341, 345 (guanacaste)

Inga edulis Mart. 245, 341 (cuajiniquil)

Inga mortoniana J.León 245 (guaba maría)

Inga oerstediana Benth. ex Seem. 245 (cuajiniquil)

Inga stenophylla Standl. 349

Inga thibaudiana DC. 349

Inga vera Willd. (= I. spuria Humb. \& Bonpl. ex Willd.)
245 (cuajiniquil)

Inga sp. 63 (cuajiniquil)

Lysiloma divaricatum (Jacq.) J.F.Macbr. (= L. seemannii Britton \& Rose) 245 (quebracho)

Mimosa diplotricha C.Wright ex Sauv. (= M. invisa Mart. ex Colla) 341, 377

Mimosa pigra L. 343 (zarza)

Pithecellobium dulce (Roxb.) Benth. 345 (michigüiste)

Prosopis juliflora (Sw.) DC. [= P. chilensis (Molina) Stuntz] 245

Samanea saman (Jacq.) Merr. [Pithecellobium saman (Jacq.) Benth.] 140 (cenízaro)

Zygia longifolia (Humb. \& Bonpl.) Britton \& Rose [Pithecellobium longifolium (Humb. \& Bonpl.) Standl.] 140, 343 (sotacaballo)

Subfamilia Papilionoideae

Calopogonium caeruleum (Benth.) Sauv. 343 (gallinita)

Canavalia rosea (Sw.) DC. (= C. maritima Thouars) 345 (frijol de playa)

Cracca mollis (Kunth) Benth. \& Oerst. [= C. micrantha Micheli, Benthamantha mollis var. micrantha (Micheli) Standl.] 369

Crotalaria incana L. 245, 349 (quiebraplato)

Crotalaria mysorensis Roth 341

Dalbergia retusa Hemsl. 245 (cocobola)

Dalbergia retusa Hemsl. var. hypoleuca (Pittier) Rudd (=D. hypoleuca Pittier) 349 (cocobola)

Erythrina berteroana Urb. 341 (poró)

Erythrina costaricensis Micheli 148, 243, 245, 349 (poró)

Erythrina fusca Lour. (= E. glauca Willd.) 349 (poró)

Gliricidia sepium (Jacq.) Kunth ex Walp. 140, 166, 177, 231, 348, 358, 370 (madero negro)

Glycine $\max$ (L.) Merr. 109 (soya, frijol de soya)

Indigofera lespedezioides Kunth (=I. mucronata Willd. ex Spreng., nom. inval.) 343

Lonchocarpus atropurpureus Benth. 369 (chaperno)

Lonchocarpus costaricensis (Donn.Sm.) Pittier 343 (pavilla)

Lonchocarpus heptaphyllus (Poir.) DC. [= L. latifolius Kunth ex DC., L. pentaphyllus (Poir.) Kunth ex DC.] 345 (chaperno)

Lonchocarpus macrophyllus Kunth $(=$ L. lucidus Pittier) 369

Lonchocarpus salvadorensis Pittier [antes se usó el nombre L. sericeus (Poir.) Kunth ex DC. var. glabrescens Benth., nom illeg.] 345 (chaperno)

Lonchocarpus sp. (= Derris sp.) 285

Lupinus aschenbornii S.Schauer 343 
Machaerium aff. floribundum Benth. 140 (bejuco de danta)

Mucuna aff. andreana Micheli 140 (ojo de buey)

Myrospermum frutescens Jacq. 245, 349

Myroxylon balsamum (L.) Harms 276 (chirraca, bálsamo de Perú)

Pachyrhizus erosus (L.) Urb. (= P. angulatus Rich. ex DC.) 12, 167 (jícama)

Phaseolus vulgaris L. 63, 340, 391 (frijol)

Piscidia carthagenensis Jacq. 349 (pellejo de toro)

Pterocarpus hayesii Hemsl. 345 (sangre de drago)

Swartzia cubensis (Britton \& P.Wilson) Standl. 245

Ulex europaeus L. 348 (tojo)

Zornia diphylla (L.) Pers. 185 (zornia, trencilla)

Fagaceae

Quercus brenesii Trel. 349 (roble)

Quercus copeyensis C.H.Mull. 245 (roble, r. blanco)

Quercus costaricensis Liebm. (= Q. irazuensis Kuntze 345) (roble)

Quercus seemannii Liebm. (=Q. borucasana Trel., Q. citrifolia Liebm., Q. eugeniaefolia Liebm.) 245, 345, 349 (roble, encino)

Flacourtiaceae

Abatia parviflora Ruiz \& Pav. 343

Casearia arguta Kunth 343 (palo maría, huesillo)

Casearia guianensis (Aubl.) Urb. 349

Laetia thamnia L. 245

Xylosma flexuosum (Kunth) Hemsl. (= X. seemannii

Triana \& Planch.) 140, 245, 349 (espino, peipute)

Zuelania guidonia (Sw.) Britton \& Millsp. 245 (plomillo)

Gesneriaceae

Achimenes erecta (Lam.) H.P.Fuchs $[=$ A. pulchella (L'Her.) Hitchc.] 140

Besleria standleyi C.V.Morton 348

Kohleria spicata (Kunth) Oerst. 343

Moussonia strigosa (C.V.Morton) Wiehler (= Kohleria strigosa C.V.Morton) 343

Grossulariaceae ( Saxifragaceae)

Escallonia myrtilloides L.f. (= E. poasana Donn. Sm.) 349 (cipresillo)

Hernandiaceae

Gyrocarpus jatrophifolius Domin (= G. americanus Jacq.) 245 (bailarina, volador)

Hydrangeaceae ( Saxifragaceae)

Hydrangea macrophylla (Thunb.) DC. (= H. opuloides
K.Koch) 345 (hortensia)

Hydrophyllaceae

Wigandia urens (Ruiz \& Pav.) Kunth (= W. caracasana Kunth) 321, 345 (ortiga de montaña)

Hypericaceae (según algunos autores: Clusiaceae $=$ Guttiferae)

Hypericum silenoides Juss. 348

Icacinaceae

Calatola costaricensis Standl. 158 (erepe, palo de papa)

Citronella costaricensis (Donn.Sm.) R.A.Howard (= Villaresia costaricensis Donn.Sm.) 349

Juglandaceae

Alfaroa costaricensis Standl. 245, 349 (gaulín)

Lamiaceae (= Labiatae)

Hyptis suaveolens (L.) Poit. 121 (chan)

Lepechinia schiedeana (Schltdl.) Vatke 87

Mentha spp. 302, 405 (menta, hierba buena, yerbabuena)

Ocimum basilicum L. 337 (albahaca)

Ocimum micranthum Willd. 147 (agua florida, albahaca)

Origanum vulgare L. 147 (orégano)

Rosmarinus officinalis L. 170 (romero)

Satureja viminea L. 47, 120 (palo de menta, menta de palo)

Scutellaria costaricana H.Wendl. 140 (pavoncillo rojo)

Thymus vulgaris L. 119, 170 (tomillo)

\section{Lauraceae}

Cinnamomum cinnamomifolium (Kunth) Kosterm. [Phoebe cinnamomifolia (Kunth) Nees] 371, 372 (aguacatillo)

Cinnamomum hammelianum (W.C.Burger) LoreaHern. (Phoebe hammeliana W.C.Burger) 284 (aguacatillo)

Licaria triandra (Sw.) Kosterm. [= L. limbosa (Ruiz \& Pav.) Kosterm.] 245 (aguacatillo)

Nectandra spp. 184, 349

Nectandra membranacea (Sw.) Griseb. 156, 318, 319 (quizarrá, ira)

Nectandra ramonensis Standl. 349

Ocotea endresiana Mez 349

Ocotea holdridgeiana W.C.Burger 336 (aguacatillo baboso)

Ocotea nicaraguensis Mez 140 
Ocotea pittieri (Mez) van der Werff (Phoebe pittieri Mez) 68, 345 (aguacatillo)

Ocotea pretiosa Benth. \& Hook.f. 140

Ocotea veraguensis (Meisn.) Mez 345 (canelo)

Ocotea spp. 184, 349

Persea albida Kosterm. (= P. pallida Mez \& Pittier, nom. illeg.) 349

Persea americana Mill. 341 (aguacate)

Persea caerulea (Ruiz \& Pav.) Mez 349 (aguacatillo, a. ascá)

Linaceae

Linum usitatissimum L. 121 (lino, linaza)

Loasaceae

Nasa speciosa (Donn.Sm.) Weigend (Loasa speciosa Donn.Sm.) 20, 26

Nasa triphylla (Juss.) Weigend (Loasa triphylla Juss.) 26, 321 (pringa-moza)

Loganiaceae

Spigelia anthelmia L. 247 (lombricera)

Loranthaceae

Gaiadendron punctatum (Ruiz \& Pav.) G.Don (= G. poasense Donn.Sm.) 348

Magnoliaceae

Magnolia poasana (Pittier) Dandy 163, 343 (magnolia, candelillo)

Malpighiaceae

Byrsonima crassifolia (L.) Kunth 341, 409 (nance)

Malpighia aff. lundellii C.V.Morton 245

Malvaceae

Abelmoschus esculentus (L.) Moench (Hibiscus esculentus L.) 293 (okra, café ñanjú)

Gossypium hirsutum L. 160 (algodón)

Gossypium mexicanum Tod. 141 (algodón)

Gossypium sp. 341 (algodón)

Hibiscus pernambucensis Arruda ( $=H$. tiliaceus L.) 343 (majagua)

Malvaviscus palmanus Pittier \& Donn.Sm. 341 (quesito, malvavisco)

Sida rhombifolia L. 140

Wercklea insignis Pittier \& Standl. 348

Melastomataceae

Bellucia pentamera Naudin (= B. costaricensis Cogn.) 31, 349 (papaturro agrio)

Bellucia sp. 349
Conostegia aff. bigibbosa Cogn. 349

Conostegia oerstediana O.Berg ex Triana 343 (mariquita)

Conostegia rufescens Naudin [= C. aff. puberula Cogn. (sic)] 349

Conostegia xalapensis (Bonpl.) D.Don 348 (lengua de vaca)

Miconia argentea (Sw.) DC. 245 (lengua de vaca, Santa María)

Miconia dodecandra Cogn. 345 (lengua de vaca, Santa María)

Miconia serrulata (DC.) Naudin 349

Monochaetum vulcanicum Cogn. 348, 349

Meliaceae

Guarea pterorhachis Harms (= G. turrialbana J.León) 245

Ruagea insignis (C.DC.) T.D.Penn. [=R. caoba (C.DC.) Harms] 245

Swietenia macrophylla King 245, 349 (caoba)

Trichilia pleeana (A.Juss.) C.DC. 349 [la obra citada indica "T. cipo (A.Juss.) C.DC.", que es una sp. de Amazonia; en Costa Rica, este último nombre se ha usado erróneamente]

Trichilia havanensis Jacq. 114, 341 (uruca)

Trichilia hirta L. 349

Trichilia martiana C.DC. (= T. cuneata Radlk.) 343

Trichilia trifolia L. 245

Menispermaceae

Cissampelos pareira L. 168, 301, 303, 341 (pareira brava)

Monimiaceae ( Siparunaceae)

Nota: La taxonomía de Siparuna es difícil; así, algunos de los nombres siguientes son dudosos.

Siparuna cujabana (Mart.) A.DC. 140 (hierba de danta; no es de Costa Rica)

Siparuna grandiflora (Kunth) Perkins (=S. tonduziana Perkins) 140,188

Siparuna griseo-flavescens Perkins 30, 140, 193, 345 (limoncillo) [probablemente se trata de $S$. gesnerioides (Kunth) A.DC.]

Siparuna guianensis Aubl. 140 (hierba del pasmo)

Siparuna paralleloneura Perkins 245, 349

Siparuna pauciflora (Beurl.) A.DC. 189

Moraceae

Artocarpus altilis (Parkinson) Fosberg $[=A$. communis J.R.Forst. \& G.Forst., A. incisa (Thunb.) L.f.] 16, 141, 317, 341, 412 (fruta de pan)

Brosimum alicastrum Sw. (= B. terrabanum Pittier) 


\section{4, 349}

Brosimum costaricanum Liebm. 245 (ojoche)

Brosimum utile (Kunth) Oken 345, 354 (mastate, árbol vaca, vaco)

Castilla elastica Sessé ex Cerv. (= C. costaricana Liebm.) 245 (hule, palo de hule)

Ficus americana Aubl. [=F. oerstediana (Miq.) Miq.] 349

Ficus colubrinae Standl. 349

Ficus costaricana (Liebm.) Miq. 341 (higuerón)

Ficus insipida Willd. (= F. glabrata Kunth) 140, 403 (higuerón)

Ficus jimenezii Standl. 140, 245, 349, 403 (higuerón, chilamate)

Ficus morazaniana W.Burger 349 [1a obra citada indica "F. lapathifolia (Liebm.) Miq.", que no es de Costa Rica. En el Herbario de la Universidad de Costa Rica (USJ) hay ejemplares de $F$. morazaniana que antes fueron determinados erróneamente como $F$. lapathifolia $]$

Ficus pertusa L.f. (=? F. padifolia Kunth) 403 (higuito)

Ficus velutina Humb. \& Bonpl. ex Willd. 245, 349 (higuerón)

Ficus sp. 140

Trophis racemosa (L.) Urb. 349

Myricaceae

Myrica cerifera L. [= Morella cerifera (L.) Small] 245, 349, 410 (arrayán, cerillo)

Myrica sp. 1

Myristicaceae

Compsoneura sprucei (A.DC.) Warb. 345 (cerezo, sangre)

Myristica fragrans Houtt. (= M. moschata Thunb., M. officinalis L.f.) 37 (nuez moscada)

Myrsinaceae

Ardisia pleurobotrya Donn.Sm. 348

Myrsine pittieri (Mez) Lundell (Rapanea pittieri Mez) 349

Stylogyne turbacensis (Kunth) Mez ssp. laevis (Oerst.) Ricketson \& Pipoly $[=$ S. ramiflora (Oerst.) Mez $] 345$ (guastomate)

Myrtaceae

Eucalyptus globulus Labill. 309 (eucalipto)

Eugenia cartagensis O.Berg. 345, 349 (murta, turrú, guayabillo)

Eugenia salamensis Donn.Sm. [= E. salamensis var. rensoniana (Stand1.) McVaugh] 245 (guayabillo)

Eugenia spp. 169, 349 (murta)
Melaleuca decora (Salisb.) Britten 172 (corcho)

Melaleuca leucadendra (L.) L. (= M. leucadendron L.) 172 (corcho, cayeput)

Ugni myricoides (Kunth) O.Berg. [= Myrtus oerstedii (O.Berg.) Hemsl.] 348

Pimenta dioica (L.) Merr. (= P. officinalis Lindl.) 140, 373, 374, 375 (canela de monte)

Psidium guajava L. 29, 55 (guayabo)

Psidium savannarum Donn.Sm. 94 (güísaro)

Psidium spp. 94

Syzygium jambos (L.) Alston (Eugenia jambos L.) 331, 341, 398 (manzana rosa)

Oleaceae

Ligustrum lucidum Aiton 343 (trueno)

Ligustrum vulgare L. 349 (olivo, o. de cerca)

Olea europaea L. 109 (olivo)

Onagraceae

Hauya lucida Donn.Sm. \& Rose [H. elegans ssp. lucida (Donn.Sm. \& Rose) P.H.Raven \& Breedlove] 349

Ludwigia erecta (L.) H.Hara (Jussiaea erecta L.) 245

Oxalidaceae

Averrhoa carambola L. 226 (carambola)

Oxalis corniculata L. 165 (acedera)

Papaveraceae

Argemone mexicana L. 162, 178, 341 (cardosanto amarillo)

Bocconia frutescens L. 140, 348, 377 (guacamaya)

Passifloraceae

Passiflora adenopoda DC. 346 (pococa, granadilla)

Phytolaccaceae

Phytolacca icosandra L. 341, 349 (caladú, jaboncillo)

Piperaceae

Piper aduncum L. (= Piper celtidifolium Kunth) 89, 140, 309 (cordoncillo)

Piper amalago L. [ $=$ P. medium Jacq., $P$. amalago var. medium (Jacq.) Yunck.] 146 (alcotán)

Piper auritum Kunth 69, 123 (hoja de estrella, anisillo, hoja de anís)

Piper darienense C.DC. 140, 168

Piper fimbriulatum C.DC. (= P. neurostachyum C.DC.) 348

Piper hispidum Sw. (= P. gonagricum Trel.) 341

Piper jaborandi Vell. 140 (no habita en Costa Rica)

Piper lanceifolium Kunth (= P. pseudolanceaefolium Trel.) 348 
Piper marginatum Jacq. (= P. marginatum var. clausum

Yunck.) 339 (hoja de estrella, anisillo)

Piper nigrum L. 210 (pimienta, p. negra)

Piper sp. 140

Pothomorphe peltata (L.) Miq. [Lepianthes peltata (L.) Raf., Piper peltatum L.] 88

Pothomorphe umbellata (L.) Miq. [Lepianthes umbellata (L.) Ramamoorthy, Piper umbellatum L.] 339 (hoja de estrella)

Plantaginaceae

Plantago australis Lam. 77 (1lantén)

Plantajo major L. 112 (llantén)

Polygalaceae

Monnina costaricensis Chodat 348

Polygonaceae

Coccoloba caracasana Meisn. 345 (papaturro blanco)

Coccoloba padiformis Meisn. 245

Muehlenbeckia platyclada (F.Müll.) Meisn. 345 (solitaria)

Polygonum punctatum Elliott 348

Rheum officinale Baill. 46, 264 (ruibarbo)

Rheum palmatum L. 264 (ruibarbo)

Rumex acetosa L. 368 (acedera)

Rumex acetosella L. 345 (ruibarbillo)

Rumex crispus L. 165, 264, 350 (ruibarbo, romaza)

Rumex obtusifolius L. 264, 350 (ruibarbo)

Portulacaceae

Portulaca oleracea L. 165, 305 (verdolaga)

Proteaceae

Grevillea robusta A.Cunn. 348 (gravilia)

Ranunculaceae

Delphinium ajacis L. [= Consolida ajacis (L.) Schur] 102 (espuela de caballero)

Rhamnaceae

Gouania polygama (Jacq.) Urb. 349

Rhamnus capreifolia Schltdl. 349

Rhamnus purshiana DC. 46 (cáscara sagrada; no habita en Costa Rica)

Ziziphus guatemalensis Hemsl. 245, 349

Ziziphus mauritiana Lam. 108, 213 (guinda, yuyuba)

Rhizophoraceae

Rhizophora mangle L. 25 (mangle)

Rosaceae

Eriobotrya japonica (Thunb.) Lindl. 341 (níspero)
Hesperomeles heterophylla (Ruiz \& Pav.) Hook. 245 (espino)

Holodiscus argenteus (L.f.) Maxim. 345

Rubus irasuensis Liebm. 343 (mora)

Rubiaceae 386

Amaioua corymbosa Kunth 349

Antirhea sp. 349

Calycophyllum candidissimum (Vahl) DC. 348 (madroño)

Chimarrhis latifolia Standl. 349 (yema de huevo)

Chimarrhis parviflora Standl. 349 (pejibayito)

Chione sylvicola (Standl.) W.C.Burger $(=C$. allenii L.O.Williams, C. costaricensis Standl.) 349

Chomelia microloba Donn.Sm. [= Anisomeris microloba (Donn.Sm.) Standl.] 349

Chomelia spinosa Jacq. 345 (malacahuite)

Chomelia sp. 349 (malacahuite)

Cinchona pubescens Vahl 46, 345 (quina)

Coffea arabica L. 95, 182, 293, 349 (café, cafeto)

Cosmibuena grandiflora (Ruiz \& Pav.) Rusby 386

Cosmibuena valerii (Stand1.) C.M.Taylor (Hillia valerii Stand1.) 349 (azaharcillo)

Coussarea sp. 349 (la obra citada indica "C. taurina Standl. \& L.O.Williams", un nombre que no aparece publicado)

Duroia costaricensis Standl. 349

Exostema caribaeum (Jacq.) Roem. \& Schult. 245, 349

Exostema mexicanum A.Gray 245, 349

Genipa americana L. [= G. americana L. var. caruto (Kunth) K.Schum.] 343 (guaitil)

Gonzalagunia panamensis (Cav.) K.Schum. 349, 386

Guettarda macrosperma Donn.Sm. 245

Guettarda poasana Standl. 349

Guettarda sp. 349

Hamelia patens Jacq. 112, 341 (zorrillo real)

Hoffmannia congesta (Oerst.) Dwyer (Xerococcus congestus Oerst.) 349

Isertia haenkeana DC. 343

Ixora coccinea L. 345 (jazmín)

Ladenbergia brenesii Standl. 349 (quina)

Lindenia rivalis Benth. 343 (lirio, jazmincillo)

Nertera granadensis (Mutis ex L.f.) Druce (Gomozia granadensis Mutis ex L.f.) 345

Pentagonia donnell-smithii (Standl.) Standl. 349

Pentagonia macrophylla Benth. 349

Pentagonia tinajita Seem. [= P. gymnopoda (Standl.) Stand1.] 349

Pogonopus speciosus (Jacq.) K.Schum. 349

Psychotria carthagenensis Jacq. 245

Psychotria emetica L.f. 140 
Psychotria ipecacuanha (Brot.) Stokes [Cephaelis ipecacuanha (Brot.) A.Rich., Uragoga ipecacuanha (Brot.) Baill.] 46, 138, 251, 277, 310 (ipecacuana, raicilla)

Psychotria poeppigiana Müll.Arg. $[=$ Cephaelis tomentosa (Aubl.) Vahl] 349

Psychotria suerrensis Donn.Sm. 240

Psychotria sp. 349

Rondeletia amoena (Planch.) Hemsl. 349 (teresa)

Rondeletia aspera Standl. 349

Rondeletia brenesii Standl. 349

Rondeletia costaricensis Standl. 349

Rondeletia reflexa Benth. [Arachnothryx reflexa (Benth.) Planch.] 349 (sp. sudamericana que, aparentemente, no habita en Costa Rica)

Schradera costaricensis Dwyer 349 (el artículo indica "S. polycephala DC.", una sp. sudamericana que no habita en Costa Rica)

Simira maxonii (Standl.) Steyerm. [Sickingia maxonii (Standl.) Standl.] 67, 349 (guaitil colorado)

Sommera donnell-smithii Standl. 349

Tocoyena pittieri (Standl.) Standl. 349

\section{Rutaceae}

Nota: En el género Citrus existe una notable confusión; según autores recientes, varios de los nombres más utilizados (aquí con $*$ ) corresponden a variedades o híbridos de C. maxima (Burm. ex Rumph.) Merr.

Casimiroa edulis La Llave \& Lex. 129, 136, 341 (matasano)

*Citrus aurantifolia (Christm.) Swingle (=? C. acida Pers.) 389 (limón agrio criollo)

*Citrus aurantifolia (Christm.) Swingle 389 (limón mandarino, 1. pangpur, 1. rangpur)

*Citrus aurantifolia (Christm.) Swingle 380 (lima, cultivar mesina)

*Citrus aurantifolia (Christm.) Swingle (=? Citrus medica L. var. acida, =? Citrus limonum Risso) 309 (limón ácido, 1. agrio)

*Citrus aurantium L. 389 (naranja agria)

*Citrus grandis (L.) Osbeck 39, 389 (toronja, grape fruit)

*Citrus limetta Risso 174, 389 (limón dulce)

Citrus medica L. var. limon L. [= Citrus limonia (L.) Osbeck, comb. inval.] 389, 404 (lima agria, limón agrio, limón rugoso)

*Citrus nobilis Lour. 389 (mandarina)

*Citrus sinensis (L.) Osbeck 4, 39, 289, 389 (naranjo, naranja dulce)

Citrus spp. 117, 388 (cítricos)

Galipea granulosa Kallunki 183

Pilocarpus racemosus Vahl 307 (talcacao)
Ruta chalepensis L. 170 (ruda)

Zanthoxylum caribaeum Lam. (= Z. elephantiasis Macfad.) 349 (lagartillo)

Zanthoxylum limoncello Planch. \& Oerst. 345 (limoncillo, zorrillo)

Zanthoxylum rhoifolium Lam. (= Z. microcarpum Griseb.) 343 (lagarto blanco)

Zanthoxylum riedelianum Engl. $(=Z$. kellermanii P.Wilson) 245 (lagartillo)

Salicaceae

Salix humboldtiana Willd. (= S. chilensis Molina, nom . rejic.) 348 (sauce)

Sapindaceae

Blighia sapida K.D.König 2, 49, 345 (seso vegetal)

Cupania glabra Sw. 341 (cascuá)

Cupania guatemalensis (Turcz.) Radlk. 343 (huesillo, pozolillo)

Dilodendron costaricense (Radlk.) A.H.Gentry \& Steyerm. (Dipterodendron costaricense Radlk.) 349 (iguano)

Dodonaea viscosa (L.) Jacq. 343

Melicoccus bijugatus Jacq. (= Melicoccus bijuga L.) 348 (mamón)

Paullinia costaricensis Radlk. 343 (hoja de pájaro)

Sapindus saponaria L. 213 (jaboncillo, chumico)

Sapotaceae

Chrysophyllum argenteum Jacq. $(=C$. panamense Pittier) 345

Chrysophyllum cainito L. 187, 341 (caimito)

Manilkara chicle (Pittier) Gilly 245, 349 (níspero) [este es el nombre científico correcto de la sp. que habita en Ciudad Colón, no M. spectabilis (Pittier) Standl.]

Manilkara zapota (L.) P.Royen [Achras zapota L., M. achras (Mill.) Fosberg] 58, 245, 349 (níspero, n. de Guanacaste, zapotillo)

Pouteria sp. 349

Pouteria campechiana (Kunth) Baehni 245, 349 (zapote)

Pouteria sapota (Jacq.) H.E.Moore \& Stearn [= Calocarpum mammosum (L.) Pierre] 49, 179 (zapote, z. colorado)

Sideroxylon persimile (Hemsl.) T.D.Penn. ssp. persimile (= Bumelia austin-smithii Standl., Bumelia panamensis Standl.) 245, 349

Scrophulariaceae

Hemichaena fruticosa Benth. 349

Scoparia dulcis L. 213, 363 (escobilla blanca, mastuerzo) 
Simaroubaceae

Ailanthus altissima (Mill.) Swingle 330 (árbol de los dioses) (esta sp. no habita ni se cultiva en Costa Rica)

Quassia amara L. 27, 134, 202, 203, 215, 253, 255, 257, 345, 399, 402 (hombre grande, cuasia)

Simarouba glauca DC. 345 (aceituno)

Solanaceae

Acnistus arborescens (L.) Schltdl. 123, 341 (güitite)

Brugmansia arborea (L.) Lagerh. (Datura arborea L.) 38, 123, 295, 341, 377 (reina de la noche)

Brugmansia candida Pers. [B. x candida Pers., Datura candida (Pers.) Saff.] 38, 123 (reina de la noche)

Cestrum aurantiacum Lindl. (= C. warszewiczil Klotzsch) 140, 343, 349 (zorrillo, pavoncillo)

Cestrum nocturnum L. 140 (zorrillo)

Cestrum sp. 140 (zorrillo)

Datura stramonium L. 51, 356, 377 (tapate, estramonio)

Schultesianthus megalandrus (Dunal) Hunz. [Markea megalandra (Dunal) D'Arcy, M. leucantha Donn. Sm.] 190

Solanum americanum Mill. (= Solanum nigrum L.) 7 , 123

Solanum capsicoides All. (= S. ciliatum Lam.) 7, 296 (naranjilla de color, huevo de perro)

Solanum dotanum C.V.Morton \& Standl. 348

Solanum hartwegii Benth. ( S. laurifolium Mill.) 7

Solanum hirtum Vahl 7

Solanum hispidum Pers. 7

Solanum lycopersicum L. (= Lycopersicon esculentum Mill.) 313 (tomate)

Solanum mammosum L. 7, 100 (pichichío)

Solanum melongena L. (= S. esculentum Dunal) 63, 127 (berenjena)

Solanum nudum Dunal (= S. parcebarbatum Bitter) 7 , 348

Solanum pulverulentum Pers. (= S. storkii C.V.Morton \& Stand1.) 7, 343

Solanum quitoense Lam. 209, 341 (naranjilla)

Solanum rudepannum Dunal $[=S$. ochraceoferrugineum (Dunal) Fernald, comb. superfl.] 7, 348

Solanum sessiliflorum Dunal (= S. topiro Dunal) 205

Solanum torvum Sw. (= S. ferrugineum Jacq.) 7, 341

Solanum tuberosum 313 (papa, patata)

Solanum umbellatum Mill. 7, 245 (zorrillo)

Witheringia asterotricha (Standl.) Hunz. (= Capsicum "aff." multiflorum Standl. \& C.V.Morton) 140

Witheringia solanacea L'Her. (= Capsicum tetramerum Standl. \& C.V.Morton) 53, 140 (sulfatillo)
Staphyleaceae

Turpinia occidentalis (Sw.) Don 343

Sterculiaceae

Guazuma ulmifolia Lam. 41 (guácimo)

Helicteres guazumifolia Kunth 345 (rabo de chancho)

Herrania purpurea (Pittier) R.E.Schult. 345 (cacao de mono, c. de monte)

Melochia pyramidata L. 341, 347, 348, 377

Theobroma cacao L. 211 (cacao)

Styracaceae

Styrax argenteus C.Presl 245 (ascá, laurel, resina)

Symplocaceae

Symplocos brenesii Standl. 348

Theaceae

Cleyera theioides (Sw.) Choisy (=C. theaeoides) 349

Freziera candicans Tul. 245, 349

Theophrastaceae

Jacquinia nervosa C.Presl (= J. macrocarpa Cav.) 345 (burriquita)

Tiliaceae

Apeiba tibourbou Aubl. 345 (peine de mico)

Corchorus orinocensis Kunth 348

Goethalsia meiantha (Donn.Sm.) Burret 349 (guácimo blanco)

Luehea candida (DC.) Mart. 343 (guácimo molenillo)

Luehea seemannii Triana \& Planch. 345 (guácimo colorado, g. macho)

Luehea speciosa Willd. 343 (guácimo macho)

Muntingia calabura L. 343 (capulín) (género incluido por algunos autores en Muntingiaceae)

Trichospermum galeottii (Turcz.) Kosterm. 245 [el nombre T. mexicanum (DC.) Baill. se ha usado inapropiadamente]

Ulmaceae

Trema micrantha (L.) Blume 349 (jucó)

Urticaceae

Phenax angustifolius (Kunth) Wedd. 304

Phenax spp. 93

Urera baccifera (L.) Gaudich. 26, 28, 321 (ortiga)

Urera caracasana (Jacq.) Griseb. 379

Urtica leptophylla Kunth 26

Verbenaceae

Citharexylum donnell-smithii Greenm. 348 (dama) 
Citharexylum viride Moldenke 345

Cornutia pyramidata $\mathrm{L}$. [= C. grandifolia $($ Schltdl. \& Cham.) Schauer] 345 (pavilla)

Lippia alba (Mill.) N.E.Br. 47 (juanilama)

Lippia berlandieri Schauer 147 (orégano)

Lippia torresii Standl. 345 (caragra)

Rehdera trinervis (S.F.Blake) Moldenke 245, 349 (yayo)

Verbena litoralis Kunth 62, 70, 71, 382 (verbena)

Vitex cooperi Standl. 345 (cuajada)

Vitaceae

Cissus verticillata (L.) Nicolson \& C.E.Jarvis $(=C$. sicyoides L.) 349 (iasú, bejuco ubi)

Vochysiaceae

Vochysia ferruginea Mart. 345 (palo de mayo, mayo)

Winteraceae

Drimys granadensis L.f. 82, 348 (chile muelo)

\section{MONOCOTILEDÓNEAS}

Agavaceae ( Liliaceae)

Yucca guatemalensis hort. ex Baker $(=$ Y. elephantipes Regel) 159, 341 (itabo)

Alstroemeriaceae ( Amaryllidaceae)

Bomarea costaricensis Kraenzl. 343 (papa de venado)

Araceae

Colocasia esculenta (L.) Schott 11 (ñampí)

Dieffenbachia seguine (Jacq.) Schott 18, 348 (sainillo)

Dracontium pittieri Engl. 140 (terciopelo, planta de culebra, comida de culebra)

Dracontium sp. 140 (la obra citada indica " $D$. dubium Kunth", pero esta sp. fue descrita de Guyana y no habita en Costa Rica)

Monstera adansonii Schott (= M. friedrichsthalii

Schott) 245 (piñanona, ventanilla)

Philodendron radiatum Schott 245

Xanthosoma roseum Schott 213 (pata, pico de pato)

Xanthosoma violaceum Schott 11, 213 (tiquisque)

Arecaceae $(=$ Palmae $)$

Acrocomia aculeata (Jacq.) Lodd. ex Mart. (= A. vinifera Oerst.) 17 (coyol, palma de coyol)

Attalea rostrata Oerst. [= A. butyracea (Mutis ex L.f.)

Wess.Boer, Scheelea rostrata (Oerst.) Burret] 383 (palma real, corozo, gunzo)

Bactris gasipaes Kunth [= Guilielma gasipaes (Kunth)

L.H.Bailey] 50, 140, 155, 392, 414 (pejibaye)
Prestoea longepetiolata (Oerst.) H.E.Moore (Euterpe longepetiolata Oerst.) 42 (palmito, pacaya de ratón)

Geonoma interrupta (Ruiz \& Pav.) Mart. (= G. binervia Oerst., G. edulis H.Wendl. ex Spruce) 376 (súrtuba amarga)

Bromeliaceae

Ananas comosus (L.) Merr. 365, 366 (piña)

Bromelia pinguin L. 275 (piñuela)

Dioscoreaceae

Dioscorea alata L. 213 (ñame, ñ. blanco)

Liliaceae ( Alliaceae)

Allium cepa L. 19, 117, 313 (cebolla)

Allium sativum L. 117, 337 (ajo)

Marantaceae

Maranta arundinacea L. 397 (sagú)

\section{Musaceae}

Musa acuminata Colla (= M. cavendishii Lamb. ex Paxton) 96 (banano verde)

Musa $x$ paradisiaca L. [= M. sapientum L., M. $x$ paradisiaca var. sapientum (L.) Kuntze] 98, 107, 173, 313, 378, 381 (banano)

Musa sp. 201 (guineo)

Orchidaceae

Oncidium aff. cebolleta Sw. 140 (lluvia de oro, cola de rata)

Poaceae (= Gramineae)

Bambusa vulgaris Schrad. 245 (bambú)

Chusquea subtessellata Hitchc. 348 (bambú)

Cymbopogon citratus (DC.) Stapf 57, 120, 140 (zacate de limón, hierba limón)

Hordeum vulgare L. 291 (cebada)

Hyparrhenia rufa (Nees) Stapf 349 (jaragua)

Oryza sativa L. 63 (arroz)

Saccharum officinarum L. 175 (caña de azúcar)

Vetiveria zizanioides (L.) Nash [Chrysopogon zizanioides (L.) Roberty] 57 (zacate violeta)

Zea mays L. 63, 109, 392 (maíz)

\section{Smilacaceae}

Smilax vanilliodora $\mathrm{Apt}(=$ S. chiriquensis C.V.Morton) 222, 223, 252 (zarzaparrilla, saskecha)

Smilax spp. 60, 140, 143, 176, 242, 401 (zarzaparrilla, cuculmeca) 


\section{Zingiberaceae}

Curcuma longa L. 140 (cúrcuma, yuquilla, camotillo) Elettaria cardamomum (L.) Maton 80 (cardamomo)
Hedychium coronarium J.König 236 (heliotropo blanco)

Zingiber officinale Roscoe 140 (jengibre) 